\title{
Extreme Star Formation
}

\author{
Jean L. Turner
}

—contribution from Astrophysics in the Next Decade: JWST and Concurrent Facilities, Astrophysics and Space Science Proceedings, 2009, ed. H. A. Thronson, M. Stiavelli, and A. G. G. M. Tielens-

\begin{abstract}
Extreme star formation includes star formation in starbursts and regions forming super star clusters. We survey the current problems in our understanding of the star formation process in starbursts and super star clusters-initial mass functions, cluster mass functions, star formation efficiencies, and radiative feedback into molecular clouds - that are critical to our understanding of the formation and survival of large star clusters, topics that will be the drivers of the observations of the next decade.
\end{abstract}

\section{Extreme Star Formation in the Local Universe}

Extreme star formation is the violent, luminous star formation that occurs in starbursts and luminous infrared galaxies. It is the formation of super star clusters that may eventually become globular clusters. It is the source of galactic winds and metal enrichment in galaxies. It is probably what most star formation in the universe was like several gigayears ago.

The process of star formation and its associated microphysics is most easily studied in the local universe where we can examine the process of star formation in detail. While there are regions in the Galaxy that may qualify as extreme star formation, most extreme systems are extragalactic. Advances in the study of extragalactic star formation during the next decade are likely to come from improvements in spatial resolution and sensitivity, particularly in the infrared and submillimeter parts of the spectrum. The refurbished HST, forthcoming JWST, and ground-based adaptive optics systems will make fundamental contributions to our understanding of the

Jean L. Turner

Department of Physics and Astronomy UCLA, Los Angeles CA 90095-1547 USA, e-mail: turner@astro.ucla.edu 
stellar content of extreme star forming regions. Herschel, ALMA, CARMA, Plateau de Bure, SMA, and SOFIA are the far-infrared, submillimeter, and millimeter telescopes that will deliver images and spectra of molecular gas in galaxies, enabling the study of the earliest stages of star formation, and the regulation of star formation by feedback into molecular clouds. With the subarcsecond and milliarcsecond resolutions now possible we can study the star formation process in other galaxies on the parsec spatial scales of molecular cores, young clusters, and Stromgren spheres.

This review is an attempt to distill a very active area of research on extreme star formation, covering both the stellar content and studies of the star-forming gas, and to project this research into the observations of the next decade. The field is a remarkably broad one, because in the process of star birth and cluster evolution, stars and gas are physically interrelated. The observations discussed here cover the range from ultraviolet spectroscopy of hot stars to millimeter line imaging of cold molecular clouds. The focus will be on star formation in the local universe where individual star-forming regions can be resolved, and the star formation process itself can be studied. Star formation in the early universe, where extreme star formation may have been more the norm than the exception, is covered elsewhere in this volume in contributions by Tom Abel and Alice Shapley.

\section{What constitutes extreme star formation in the local universe?}

Advances in instrumentation have shaped and refined our current view of "extreme star formation" (ESF). Many of the features of ESF in the local universe were known half a century ago: giant HII regions (Burbidge and Burbidge, 1962), galactic winds (Burbidge et al. 1964: Lynds and Sandage, 1963), young "populous clusters" (Gascoigne and Kron 1952; Hodge, 1961), O-star dominated compact dwarf galaxies (Sargent and Searle, 1970), and bright extragalactic radio sources (Weedman et al. 1981). The recognition that there were individual star formation events that could energetically dominate the evolution of a galaxy came with the development of infrared and high resolution radio capabilities (Gehrz et al., 1983: Rieke and Lebofsky, 1978, 1979, Rieke and Low, 1975). However, it was the IRAS all-sky survey in the mid and far-infrared that established the universality and energetic importance of the "starburst" to galaxy evolution. IRAS demonstrated that the luminous output of galaxies can be dominated by infrared emission and recent star formation (de Jong et al. 1984: Soifer et al. 1984, 1986, 1987a b) and that extreme star formation may even be linked to the development of nuclear activity in galaxies (Sanders et al., 1988). Many of the early IRAS results have been followed up with the subsequent ISO (Genzel and Cesarsky, 2000) and Spitzer (Lonsdale et al. 2003) infrared space observatories.

The definition of starburst has evolved since the time of IRAS. In early incarnations it described systems that would deplete their gas in substantially less than a Hubble time. However, this definition can exclude galaxies with substantial reservoirs of gas that are forming stars at prodigious rates. Infrared luminosity can be 
used to classify these extreme star-forming systems: "Ultraluminous infrared galaxies" (ULIRGs) have luminosities of $\mathrm{L}_{\mathrm{IR}}>10^{12} \mathrm{~L}_{\odot}$ (Soifer et al., 1987b) and "luminous" infrared galaxies have $\mathrm{L}_{\mathrm{IR}}>\sim 10^{11} \mathrm{~L}_{\odot}$. These luminous galaxies owe most of their energetic output to star formation (Genzel et al., 1998; Sanders et al., 1986). Another definition captures the localized intensity of starbursts: Kennicutt (1998) defines starburst in terms of a star-forming surface density, $100 \mathrm{M}_{\odot} \mathrm{pc}^{-2} \mathrm{Gyr}^{-1}$, or in terms of luminosity, $10^{38.4}-10^{39.4} \mathrm{erg} \mathrm{s}^{-1} \mathrm{kpc}^{-2}$.

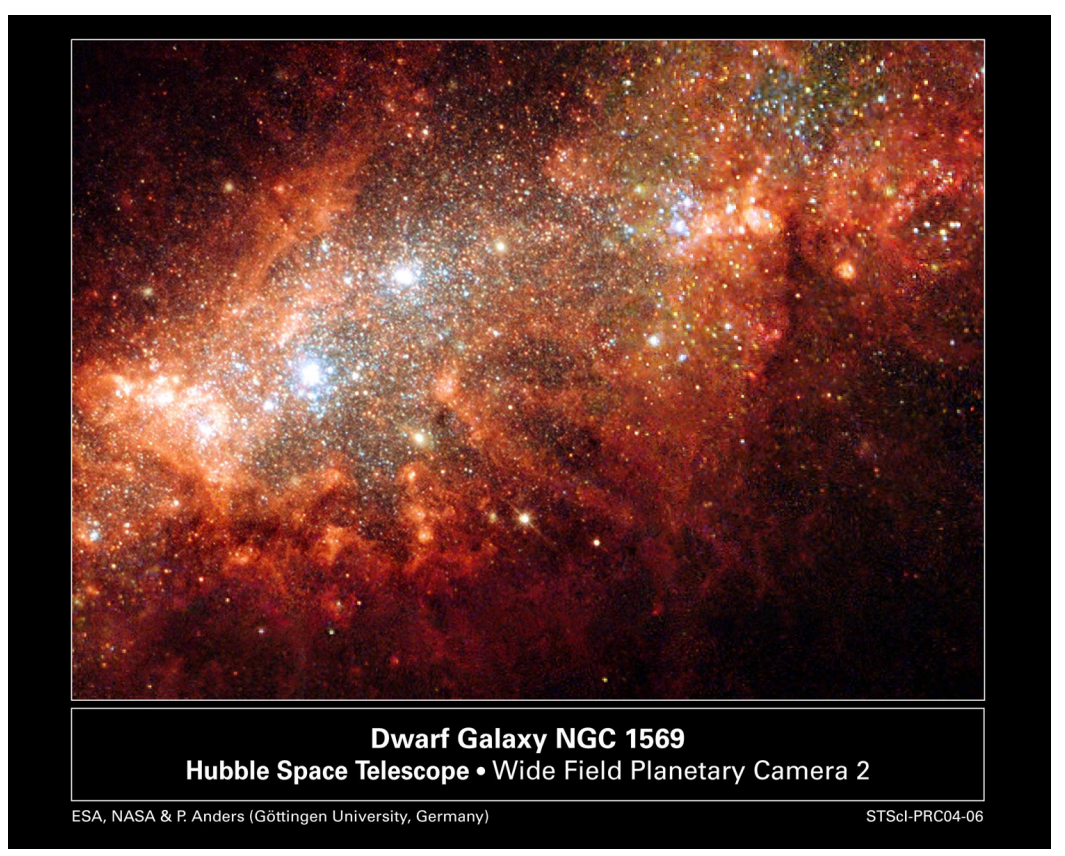

Fig. 1 HST revealed that the bright sources NGC 1569-A and NGC 1569-B were large clusters of stars. Credit: ESA, NASA, and P. Anders.

High spatial resolution has also modified our view of starbursts, revealing that they often, and perhaps nearly always, consist of the formation of large numbers of extremely large clusters, "super star clusters." For the purposes of this review, we will use the term super star cluster (SSC) to denote massive young clusters of less than $\sim 100 \mathrm{Myr}$ in age, and globular clusters to be those systems more than 7-10 Gyr in age. Both starbursts and SSCs comprise "extreme star formation."

The Hubble Space Telescope (HST) is largely responsible for the recognition of the ubiquity of SSCs and their importance in starbursts. The idea that massive clusters similar to globular clusters are actually forming in large numbers at the present time was slow to germinate, probably due to lack of spatial resolution and our inability to resolve them, although the possibility was recognized early on in the large clusters of the Magellanic clouds (Hodge, 1961). The cluster R136, the star clus- 
ter responsible for the lovely 30 Doradus Nebula in the Large Magellanic Clouds, was believed by many to be a single supermassive star before it was resolved with speckle observations from the ground (Weigelt and Baier, 1985). Other mysterious objects included two bright sources in the nearby dwarf galaxy NGC 1569, which were difficult to classify due to the uncertainty in distance to this nearby galaxy. Regarding the two "super star cluster" candidates, Arp \& Sandage (1985) stated:

A definite resolution of the present problem in NGC 1569, and for the same problem with the bright object in NGC 1705, lies in the spatial resolution into stars of these three highluminosity blue objects using the imaging instrument of the wide-field camera of Space Telescope.

HST did indeed resolve the objects in NGC 1569, revealing that they were large and luminous star clusters. In Figure 1 is shown the HST image of NGC 1569, with objects A and B referred to by Arp and Sandage. Object A consists of two superimposed clusters; crowded conditions and confusion complicate the study of SSCs, even in the closest galaxies and with the angular resolution of HST! R136 was resolved into a compact and rich cluster of stars by HST (de Marchi et al., 1993, Hunter et al., 1996). HST imaging also revealed super star clusters in NGC 1275, M82, NGC 1705, the Antennae, and numerous other starburst galaxies (Holtzman et al., 1992, Maoz et al., 1996; , Meurer et al., 1995; O'Connell et al., 1994, Whitmore and Schweizer. 1995, Whitmore et al. 1993) The discovery of young, blue star clusters with luminosities consistent with those expected for young globular clusters in local starburst galaxies meant not only that conditions favorable to the formation of protoglobular clusters exist in the present universe, but also that this extreme form of star formation is close enough for the star formation process itself to be studied.

Table 1 Super Star Clusters in Context

\begin{tabular}{lcccccc}
\hline Type & $N_{*}$ & $\begin{array}{c}\text { Mass } \\
\left(\mathrm{M}_{\odot}\right)\end{array}$ & $\begin{array}{c}\mathrm{r}_{\mathrm{h}} \\
(\mathrm{pc})\end{array}$ & $\begin{array}{c}\rho_{h}^{a} \\
\left(\mathrm{M}_{\odot} \mathrm{pc}^{-3}\right)\end{array}$ & $\mathrm{M}_{\mathrm{V}}$ & $\begin{array}{c}\text { Age } \\
(\mathrm{yrs})\end{array}$ \\
\hline globular cluster & $>10^{5}$ & $10^{3.5}-10^{6}$ & $0.3-4$ & $10^{-1}-10^{4.5}$ & -3 to -10 & $>10^{10}$ \\
open cluster & $20-2000$ & $350-7000$ & $2.5-4.5$ & $1-100$ & -4.5 to -10 & $10^{6}-10^{9.8}$ \\
embedded cluster & $35-2000$ & $350-1100$ & $0.3-1$ & $1-5$ & $\ldots$ & $10^{6}-10^{7}$ \\
SSC & $>10^{5}$ & $10^{5}-10^{6}$ & $3-5$ & $\ldots$ & -11 to -14 & $10^{6}-10^{7}$ \\
\hline
\end{tabular}

${ }^{a}$ Half mass mean density. Number of members, $N_{*}$, is not as well-defined for the larger clusters as it is for the local open and embedded clusters. References. Battinelli \& Capuzzo-Dolcetta 1991. Billett et al. 2002. Harris 1996. Harris \& Harris 2000. Lada \& Lada 2003. Mackey \& Gilmore 2003. McLaughlin \& Fall 2008. McLaughlin \& van der Marel. 2005. Noyola \& Gebhardt 2007.

Super star clusters appear to be sufficiently massive and rich to be globular clusters, differing from them only in age. Table 1 lists the general characteristics of different classes of Galactic star clusters and SSCs. The brightest young SSCs have $M_{V} \sim-14$. They are brighter than globular clusters because of their youth. It is convenient to take the lower bound for SSCs to be $M_{V} \sim-11$ (Billett et al., 2002), approximately the magnitude of R136 in the LMC, which is also comparable in size 
to the most massive young Galactic clusters. However, R136 is considered by some to be on the small side for a globular cluster. The older LMC cluster NGC 1866, at $\mathrm{L} \sim 10^{6} \mathrm{~L} \odot$ and an intermediate age of $100 \mathrm{Myr}$, is closer to a genuine globular cluster (Meylan, 1993). The upper limit to the ages of SSCs is also somewhat arbitrary; while there is evidence that typical cluster dissolution timescales are about 10

Myr, there are also intermediate age clusters with ages of $\sim 1 \mathrm{Gyr}$ even within the Local Group.

\section{Extreme Star-forming Regions of the Local Universe}

The most luminous young SSCs of the Galactic neighborhood are the testbeds for study of the star formation process in large clusters and in starburst systems. What are the Orions of the SSC world? In Table 2 we have compiled from the literature properties of some well-studied and spatially resolved SSCs in the local universe. Included are Galactic center clusters and large Galactic star-forming regions. While smaller than many extragalactic SSCs, these Galactic clusters are close and more easily studied and should share many of the star-forming properties. The super star clusters of Table 2 reflect a wide range of environments and evolutionary stages in the formation and evolution of SSCs.

The Galactic massive young clusters are readily resolved into stars and contain a wealth of information on young massive cluster evolution. However, even for these nearby clusters, confusion, contamination, and rapid dynamical evolution introduce great complexity into observational interpretations. Westerlund 1 is the closest of these large clusters, located in the Carina arm. NGC 3603 is a large southern cluster somewhat more distant. The Arches, Quintuplet, and Galactic Center nuclear clusters have formed in the immediate vicinity of a supermassive black hole, and may differ in structure and evolution from large clusters in more benign environments. The study of the Galactic Center clusters has been made possible by high resolution infrared observations. Included in Table 2 are luminous embedded star-forming regions SgrB2 and W49A; their relation to the massive, unembedded star clusters is unclear, although they have similar total luminosities. The other SSCs listed are in galaxies within $\sim 20 \mathrm{Mpc}$, in which clusters can be spatially resolved. Many of these SSCs have been identified by their location within "Wolf-Rayet" galaxies, those galaxies with a strong HeII 4686 line indicating the presence of significant numbers of Wolf-Rayet stars of age 3-4 Myr (Conti, 1991; Schaerer et al., 1999). The Wolf-Rayet feature is a relatively easy way to identify in systems with large clusters of young stars, and these are often found in SSCs. Many of the clusters in Table 2 have dwarf galaxy hosts. This may be a selection effect due to the difficulty of isolating clusters amid the higher confusion and extinctions in large spirals, since SSCs are definitely present in many local spirals, such as NGC 253, NGC 6946, and Maffei 2 (Condon, 1992; Maoz et al., 2001, 1996; Rodríguez-Rico et al. 2006, Roy et al. 2008; Tsai et al. 2006; Turner and Ho, 1994, Watson et al., 1996). The 
Table 2 Massive Young Star Clusters in the Local Universe

\begin{tabular}{llccccccccc}
\hline Host & Cluster & $\begin{array}{c}\mathrm{D} \\
(\mathrm{Mpc})\end{array}$ & $\begin{array}{c}\mathrm{R}^{a} \\
(\mathrm{pc})\end{array}$ & $\log \frac{\mathrm{L}_{*}}{\mathrm{~L}_{\odot}}$ & $\log \frac{\mathrm{M}_{*}}{\mathrm{M}_{\odot}}$ & $\log \mathrm{N}_{\text {Lyc }}$ & $\mathrm{N}_{\mathrm{O}}$ & $\mathrm{M}_{\mathrm{V}}$ & $\begin{array}{c}\text { Age } \\
(\mathrm{Myr})\end{array}$ \\
\hline Galaxy & Arches & 0.008 & $>0.5$ & 8.0 & 4.1 & 51.0 & 160 & $\ldots$ & $2-2.5$ \\
Galaxy & Quintuplet & 0.008 & 1.0 & 7.5 & $3-3.8$ & 50.9 & 100 & $\ldots$ & $3-6$ \\
Galaxy & Center & 0.008 & 0.23 & 7.3 & $3-4$ & 50.5 & 100 & $\ldots$ & $3-7$ \\
Galaxy & Sgr B2 & 0.008 & 0.8 & 7.2 & $\ldots$ & 50.3 & $(100)$ & $\ldots$ & $\ldots$ \\
Galaxy & NGC 3603 & 0.0076 & 4.5 & 7.0 & 3.4 & 50.1 & $>50$ & $\ldots$ & $1-4$ \\
Galaxy & Westerlund 1 & 0.0045 & 1 & $\ldots$ & 4.7 & 51.3 & 120 & $\ldots$ & $3-4$ \\
Galaxy & W49A & 0.014 & 5 & 7.4 & $\ldots$ & 50.1 & 80 & $\ldots$ & $\ldots$ \\
LMC & R136 & 0.05 & 2.6 & $\ldots$ & 4.8 & 51.7 & $>65$ & -11 & $1-3$ \\
NGC1569 & NGC1569-A1 & 2.2 & $1.6-1.8$ & $\ldots$ & 6.11 & $\ldots$ & $\ldots$ & -13.6 & $\ldots$ \\
NGC1569 & NGC1569-A2 & 2.2 & $1.6-1.8$ & $\ldots$ & 5.53 & $\ldots$ & $\ldots$ & $\ldots$ & $\ldots$ \\
NGC1569 & NGC1569-B & 2.2 & 3.1 & $\ldots$ & 5.6 & $\ldots$ & $\ldots$ & -12.7 & $15-25$ \\
NGC 1705 & NGC1705-1 & 5.3 & 1.6 & $\ldots$ & 5.68 & $<51$ & $\ldots$ & -14.0 & 12 \\
He 2-10 & He 2-10-1 & 3.8 & 1.5 & $\ldots$ & 5.7 & $\ldots$ & 1300 & -14.3 & 5.2 \\
He 2-10 & He 2-10-A-4 & 3.8 & 3.9 & $\ldots$ & $\ldots$ & 52.4 & $\ldots$ & $\ldots$ & $\ldots$ \\
He 2-10 & He 2-10-A-5 & 3.8 & 1.7 & $\ldots$ & $\ldots$ & 51.9 & $\ldots$ & $\ldots$ & $\ldots$ \\
He 2-10 & He 2-10-B-1 & 3.8 & 1.8 & $\ldots$ & $\ldots$ & 51.9 & $\ldots$ & $\ldots$ & $\ldots$ \\
He 2-10 & He 2-10-B-2 & 3.8 & 1.8 & $\ldots$ & $\ldots$ & 52.0 & $\ldots$ & $\ldots$ & $\ldots$ \\
M82 & M82-A1 & 3.6 & 3.0 & 7.9 & 6.0 & 50.9 & 100 & -14.8 & 6.4 \\
M82 & M82-F & 3.6 & 2.8 & 7.73 & 5.8 & $\ldots$ & $\ldots$ & -14.5 & $50-60$ \\
M82 & M82-L & 3.6 & $\ldots$ & $\ldots$ & 7.6 & $\ldots$ & $\ldots$ & $\ldots$ & $\ldots$ \\
NGC3125 & NGC3125-A1 & 11.5 & $\ldots$ & $\ldots$ & $\ldots$ & $52.4^{d}$ & $250-3000^{e}$ & $\ldots$ & $3-4$ \\
NGC3125 & NGC3125-A2 & 11.5 & $\ldots$ & $\ldots$ & $\ldots$ & $\ldots$ & $550-3000$ & $\ldots$ & $3-4$ \\
NGC3125 & NGC3125-B1,2 & 11.5 & $\ldots$ & $\ldots$ & $\ldots$ & 52.2 & 450 & $\ldots$ & $3-4$ \\
Antennae & Antennae-IR & 13.3 & $<32$ & $\ldots$ & 6.48 & 52.6 & 120 & $-17^{f}$ & 4 \\
NGC4214 & NGC4214-1 & 4.1 & $<2.5$ & $\ldots$ & $\ldots$ & $\ldots$ & 280 & -13.1 & $\ldots$ \\
NGC5253 & NGC5253-5 & 3.8 & $\ldots$ & 5.8 & $\ldots$ & 51.9 & 155 & $\sim-14$ & 2 \\
NGC5253 & NGC5253-IR & 3.8 & 0.7 & 9.0 & $\ldots$ & 52.5 & $1200-6000^{g}$ & $\ldots$ & $2-3$ \\
\hline
\end{tabular}

${ }^{a}$ Cluster radii are half-light radii. ${ }^{b}$ Cluster A is two clusters, de Marchi et al. 1997. ${ }^{c}$ Bright core of a larger, complex cluster, NGC 2070. ${ }^{d} \mathrm{~A} 1$ and A2. ${ }^{e}$ Range in $\mathrm{O}$ stars is due to differences in reddening. ${ }^{f} \mathrm{M}_{\mathrm{K}}$. ${ }^{g}$ Resolved source; lesser number for $r<1$ pc. References. Arches, Quintuplet, Galactic nuclear center clusters: Figer et al. 1999, Lang et al. 2001, Figer et al. 2005, Stolte 2003, Stolte et al. 2002, 2005, 2007, Najarro et al. 2004, Figer 2003, 2004, 2008. Kim et al. 2000, 2004, 2007, Kim \& Morris 2003. Sgr B2: Dowell 1997, Gaume et al. 1995. NGC 3603: de Pree, Nysewander, \& Goss 1999, Eisenhauer et al. 1998, Pandey et al. 2000, Drissen et al. 2002, Nürnberger \& Petr-Gotzens 2002, Stolte et al. 2006, Harayama et al. 2008, Melena et al. 2008. W49: Smith et al. 1978, Welch et al. 1987, Conti \& Blum 2002, Homeier \& Alves 2005. Westerlund 1: Clark et al. 1998, 2005, Nürnberger et al. 2002, Nürnberger 2004, Crowther et al. 2006, Mengel \& Tacconi-Garman 2007, 2008, Brandner et al. 2008. R136: Mills et al. 1978, Meylan 1993, Hunter et al. 1995, Massey \& Hunter 1998, Noyola \& Gebhardt 2007. M82-A1: Smith et al. 2006. M82-F: Smith \& Gallagher 2001, O'Connell et al. 1995, McCrady et al. 2005. M82-L: McCrady \& Graham 2007. NGC1569 A and B: O'Connell et al. 1994, Sternberg 1998, Hunter et al. 2000, Ho \& Filippenko 1996a, Smith \& Gallagher 2001, Origlia et al. 2001, Gilbert 2002, Larsen et al. 2008. NGC1705-1: Ho \& Filippenko 1996b, Sternberg 1998, Smith \& Gallagher 2001, Johnson et al. 2003, Vázquez et al. 2004. He 2-10-1: Chandar et al. 2000. One of five clusters within He 2-10A. He 2-10-A, B: Vacca \& Conti 1992, Johnson \& Kobulnicky 2003. NGC3125: Vacca \& Conti 1992, Schaerer et al. 1999ab, Stevens et al. 2002, Chandar et al. 2004, Hadfield \& Crowther 2006. Region A has $\log Q_{0}=52.39$, for $4000 \mathrm{O}$ stars, region B $\log Q_{0}=52.19$, for $3200 \mathrm{O}$ stars, Hadfield \& Crowther. Antennae: Gilbert et al. 2000, for 13.3 Mpc. NGC5253-5: Gorjian 1996, Calzetti et al. 1997, Schaerer et al. 1997, Tremonti et al. 2001, Chandar et al. 2004, Vanzi \& Sauvage 2004, Cresci et al. 2005. NGC5253-IR: Obscured IR/radio source offset by $\sim 0.5^{\prime \prime}$ from NGC5253-5. Beck et al. 1996, Turner et al. 1998, 2000, 2003, Mohan et al. 2001, Alonso-Herrero et al. 2004, Turner \& Beck 2004, Martín-Hernandez et al. 2005, Rodríguez-Rico et al. 2007. 
dominance of dwarf galaxy hosts may also be due to "downsizing," the tendency for star formation to occur in smaller systems at later times.

It is evident from Table 2 that it can be difficult to compare these clusters because embedded and visible clusters are characterized in different ways. Embedded clusters are often characterized by photons that have been absorbed by gas or dust, with well-defined Lyman continuum fluxes and infrared luminosities. Visible clusters have star counts, cluster magnitudes, colors, and stellar velocity dispersions; these clusters can have good masses and ages. Putting together an evolutionary sequence of objects thus requires multiwavelength observations at high spectral resolution. Extinction is observed to decrease with increasing cluster age (Mengel et al. 2005), as one might expect from Galactic star-forming regions, so the embedded clusters are likely to also be the youngest clusters.

High angular resolution is key to the study of even the closest super star clusters, which are often found forming in large numbers. One of the first known super star clusters, NGC 1569-A, consists of two superimposed clusters (de Marchi et al., 1997), which is not immediately obvious even in the HST image (Figure 1). The embedded IR/radio SSC in the center of NGC 5253 was found to be offset by a fraction of an arcsecond from the brightest optical cluster, NGC 5253-5, (Calzetti et al. 1997) only 5-10 pc away (Alonso-Herrero et al., 2004; Turner et al., 2003).

Extinction can also be extreme in bright IR-identified starburst regions, and can obscure even the brightest clusters through the near-IR. Observed differential extinctions between the infrared Brackett lines at 2 and $4 \mu \mathrm{m}$ indicate $A_{V}>1$ and even $A_{K}>1$ in many starbursts (Ho et al. 1990; Kawara et al., 1989). IR-derived extinctions are often higher than those derived from Balmer recombination lines toward the same regions (Simon et al., 1979) because of extinctions internal to the HII regions themselves. In M82, near-IR and mid-infrared spectroscopy indicates extinctions of $A_{V} \sim 25$ (Simon et al., 1979;: Willner et al., 1977) to $A_{v} \sim 50$ (Förster Schreiber et al., 2001), similar to values observed in Galactic compact HII regions, but over much larger areas. The clusters in Arp 220 are heavily obscured, with estimated $A_{V} \sim 10-45 \mathrm{mag}$ (Genzel et al., 1998; Shioya et al. 2001); regions behind the molecular clouds can reach $A_{V} \sim 1000$ (Downes and Solomon, 1998).

What does the next decade hold for the clusters of Table 2 and other nearby clusters like them? First, there are more SSCs to discover in the local universe, particularly embedded ones. IRAS is still a valuable tool for discovering young SSCs, but with arcminute resolution, it is not sensitive to bright, subarcsecond sources. There undoubtedly remain many compact, young ESF events to be found in the local universe. The WISE mission, an all sky mid-IR survey, will provide an extremely valuable dataset for discovering young and embedded SSCs. The enhanced sensitivities and high spatial resolution of the next generation of telescopes (JWST, EVLA, ALMA, SOFIA), will redefine our concept of "local" SSC formation, extending this list to more distant systems and to young SSCs within large, gas-rich spirals. The near-infrared, in particular, is an valuable link between visible and embedded SSCs. Subarcsecond resolutions are necessary to resolve individual clusters in regions of high and patchy extinction, and the closest galaxies are even now being pursued with adaptive optics. JWST and future extremely large ground-based telescopes will play 
an important role in connecting embedded clusters to their older, visible siblings to enable a longitudinal study of SSC evolution.

\section{Initial Mass Functions and the Most Massive Stars in SSCs}

The initial mass functions (IMFs) of SSCs have important consequences for cluster masses, their long-term survival, and potentially, for the eventual remnants left by their dissolution. Are the IMFs of clusters power law? Is there evidence for topheavy IMFs? How do the IMFs of SSCs compare to Galactic IMFs? Is there evidence for initial mass segregation in young clusters? The most massive star in the universe is likely to be in an SSC; is there a fundamental limit to the mass of stars? There is a review of the outpouring of recent IMF work on massive young clusters by Elmegreen (2008).

Mass functions have been determined for the large star clusters of the Local Group. Many appear to be Salpeter. The Salpeter mass functions are defined as $\xi(\mathrm{M}) \sim \mathrm{M}^{-\alpha}$, where $\alpha=2.35$, or, expressed in logarithmic mass intervals as $\xi_{\mathrm{L}} \mathrm{d} \log \mathrm{M} \sim \mathrm{M}^{\Gamma} \mathrm{d} \log \mathrm{M}$, with $\Gamma=-1.35$ (Scalo, 1986, 1998). The Kroupa IMF is Salpeter at higher masses, and flattens to $\alpha=1.3$ for stars below $0.5 \mathrm{M}_{\odot}$ (Kroupa, 2001). Kroupa IMFs often cannot be distinguished from Salpeter in extragalactic SSCs. We will adopt the $\Gamma$ convention here and note that while in many cases these are referred to as IMFs, what is observed is actually a present day mass function (PDMF), from which an IMF may be inferred.

The PDMF of R136 in the LMC has been measured down to $0.6 \mathrm{M}_{\odot}$ (Massey and Hunter, 1998), where it is consistent with Salpeter, $\Gamma \sim-1.3$, and then flattens below $2 \mathrm{M}_{\odot}$ to $\Gamma \sim-0.3$ (Sirianni et al. 2000). For a Salpeter or Kroupa power law IMF, flattening or turnover of the power law corresponds to an effective "characteristic mass" for the cluster (Lada and Lada, 2003). R136 thus appears to have a characteristic mass of 1-2 $\mathrm{M}_{\odot}$, as compared to $\sim 0.5-1 \mathrm{M}_{\odot}$ for the much smaller nearby Galactic embedded clusters (Lada and Lada, 2003). The southern cluster NGC 3603 has a somewhat flatter-than-Salpeter power law slope of $\Gamma=-0.7$ to -0.9 from 0.4 to $20 \mathrm{M}_{\odot}$ (Harayama et al. 2008; Stolte et al. 2006; Sung and Bessell, 2004). Arches has a very similar power law PDMF with $\Gamma=-0.8$ down to $1.3 \mathrm{M}_{\odot}$ (Stolte et al. 2002), which may correspond to an IMF that is close to Salpeter $\Gamma=-1.0$ 1.1 (Kim et al. 2007). The Arches cluster is mass segregrated, with a flatter slope in the center, $\Gamma \sim 0$, than in the outer parts of the cluster, and the mass function may turn over at 6-7 $\mathrm{M}_{\odot}$ in the core (Stolte et al. 2005); however, the MF at larger radii does not show this effect (Kim et al., 2007). Trends appear to be toward flatter MF power laws and higher characteristic masses for the largest clusters in the Local Group, but the numbers of clusters are very small, and dynamical effects are poorly understood as yet (Stolte et al. 2002).

Beyond the Local Group, it is more difficult to determine IMFs. Observational quantities for more distant SSCs are integral properties such as total luminosity and dynamical masses from cluster velocity dispersions. Constraints on IMFs from these 
integral properties require assumptions about mass cutoffs, cluster ages, and cluster structure. There is accumulating evidence, however, that IMFs in starbursts and the IMFs in SSCs, if Salpeter, may have higher characteristic cutoffs than typical Galactic clusters. Rieke et al. (1980) modeled the starburst in M82 from its IR emission properties, and concluded that the IMF of the starburst must have a low mass cutoff of 3-8 $\mathrm{M}_{\odot}$. Sternberg (1998) used visible mass-to-light ratios to argue for a cutoff of $1 \mathrm{M}_{\odot}$ for NGC1705-1, although he does not find a cutoff for NGC 1569A. If the IMF in the M82 SSCs is Salpeter, then McCrady et al. (2003) find that the individual M82 clusters must have truncated mass functions, although some clusters show strong evidence for mass segregation and possible dynamical evolution, which complicates this interpretation (Boily et al., 2005; McCrady and Graham. 2007: McCrady et al., 2005, McMillan and Portegies Zwart 2003).

What the low mass cutoffs are for SSCs, whether this varies with environment and how, and what is the likely cause of the low mass cutoffs, effective characteristic masses, and the effects of mass segregation on cluster masses and evolution will be fertile ground for SSC research in the next decade.

At the other end of the IMF, there is the question of what is the limiting mass of a star. If the cluster IMFs bear any resemblance to the Galactic power law Salpeter function, then newly-formed clusters consisting of hundreds of thousands to millions of stars are the place to find the most massive and rare $\mathrm{O}$ stars. In addition, in dense star clusters there is the possibility of the formation of very massive stars through stellar collisions, which becomes a viable mechanism in dense environments (Bonnell et al., 1998, Clarke and Bonnell, 2008). What is the limiting mass of stars in the local universe? Where are the most massive stars found?

Spectroscopy of individual stars is the most reliable way to identify the most massive stars, but is only possible within the Local Group. Ultraviolet spectroscopy using the STIS instrument on HST has allowed the classification of 45 known stars of spectral class $\mathrm{O} 2$ and $\mathrm{O} 3$; of these, 35 are found in the LMC cluster R136 (Walborn et al., 2002). R136 alone contains more than $65 \mathrm{O}$ stars (Massey and Hunter. 1998). In addition to these visible $\mathrm{O}$ stars, there is also a significant population of infrared $\mathrm{O}$ stars and Wolf-Rayet stars in the Galactic Center, including the three large star clusters, Arches, Quintuplet, and Galactic Center. These infrared clusters, each about an order of magnitude less massive than R136, contain an estimated 360 O stars among them, nearly 60 Wolf-Rayet stars, and 2-3 LBVs (Figer. 2008).

The highest inferred stellar mass in the Local Group is $\sim 170 \mathrm{M}_{\odot}$ in the LMC, and $\sim 200 \mathrm{M}_{\odot}$ in the Galaxy; however the latter could eventually turn out to be a binary system; the largest dynamical mass measured is $90 \mathrm{M}_{\odot}$ (Walborn et al. 2002). Weidner \& Kroupa (2004) estimate that based on its IMF, observed to be Salpeter, there should be one $750 \mathrm{M}_{\odot}$ star in the R136 cluster, and instead the upper mass limit appears to be $200 \mathrm{M}_{\odot}$ (Koen, 2006). Oey \& Clarke (2005) extend this to a larger sample of OB associations, obtaining a cutoff of 120-200 $\mathrm{M}_{\odot}$, although they caution that this cutoff is sensitive to the IMF slope for stars $>10 \mathrm{M}_{\odot}$. Figer (2005) finds that there should be one star of $\mathrm{M} \sim 500 \mathrm{M}_{\odot}$ in the Galactic center, where the current upper mass limit instead seems to be $130 \mathrm{M}_{\odot}$. These observations argue for a stellar upper limit close to the observed $150-200 \mathrm{M}_{\odot}$. However, given 
the small statistics, it may be that the absence of extremely massive stars is simply an evolutionary effect (Elmegreen, 2008). For example, modeling suggests that the Pistol Star in the Galactic Center may have had an initial mass of 200-250 $\mathrm{M}_{\odot}$ (Figer et al. 1998, Najarro and Figer. 1998). Stars with masses of several hundred times solar would evolve rapidly (Yungelson et al. 2008), will lose significant fractions of their initial masses, and in addition, might be dust-enshrouded for most of their lives. Moreover small number statistics at the upper mass end mean that for most clusters, the power law slopes of IMFs are uncertain to a few tenths for clusters of the size of NGC 3603 (Elmegreen, 2008). Supermassive stars are elusive by nature.

If extremely high mass stars do exist and we have not seen them simply because of their rapid evolution, the best place to look for them is in the youngest and largest clusters. These will probably be found in starbursts. There is a good chance that the youngest regions containing the most massive stars will be deeply embedded, perhaps extinguished even in the near-infrared. In these cases, nebular diagnostics provide another way of gauging the upper end of the mass function. The mid-infrared has a number of fine structure lines that can be observed in large HII regions in external galaxies (Dale et al., 2006, Helou et al., 2000; Thornley et al., 2000), including fine structure lines of $\mathrm{Ar}, \mathrm{Ne}, \mathrm{S}$, and $\mathrm{O}$, that can provide valuable nebular diagnostics of the input stellar radiation field even in embedded sources.

Line ratios of the mid-IR nebular fine structure lines in starburst galaxies measured with the SWS spectrometer on ISO revealed unexpectedly low excitation HII regions. Nebular models of the ISO lines indicate upper mass cutoffs of $\mathrm{M}_{\text {upper }} \sim 30 \mathrm{M}_{\odot}$ for the IMFs in these starbursts (Thornley et al. 2000). Given the luminosities and inferred stellar masses, the low upper mass cutoffs are surprising. However, there are a number of possible explanations for low excitation that would allow for the presence of more massive stars (Rigby and Rieke, 2004). One of these is lack of spatial resolution. Starburst regions often have extended regions of ionized gas which, when combined with the comparatively hard spectra of the compact SSC HII regions, will tend to wash out the high excitation lines. That the lines of [OIV] $25.9 \mu \mathrm{m}$, [NeIII] $15.55 \mu \mathrm{m}$, and [SIV] $10.51 \mu \mathrm{m}$ have been detected in dwarf starbursts suggests that hot stars are indeed present in some SSCs (Beck et al., 1996, 2007; Crowther et al., 1999, 2006; Lutz et al., 1998). Strong dependences on metallicity and differences in the input radiation fields from existing stellar models that must be considered in these interpretations (Crowther et al., 1999; MartínHernández et al., 2002, Rigby and Rieke, 2004).

High spatial resolution and spectroscopy from space will allow great improvements in our knowledge of the most massive stars and the upper mass cutoffs of SSC IMFs in external galaxies in the next decade. Optical and ultraviolet emission lines including Wolf-Rayet features will continue to be important diagnostics of the high mass stellar content and ages of SSCs in an enlarged sample of sources. Access to mid-IR fine structure lines from space, via JWST, will give valuable information on the most massive stars in embedded young SSC nebulae, allowing observation of homonuclear line ratios of $\mathrm{Ar}$ and $\mathrm{Ne}$ that are not possible from the ground. The improvement in spatial resolution is also extremely important for isolating the spectral signatures of compact SSC nebulae. The MIRI IFU on JWST, with order-of- 
magnitude improvements in sensitivity and spatial resolution over previous instruments, will be an extremely powerful tool for isolating compact SSC nebulae from more diffuse and potentially lower excitation ionized gas within the galaxies. The form of the cluster IMFs is key input to the question of the long term survivability of clusters.

\section{What are the initial cluster mass functions for SSCs?}

How are SSCs related to globular clusters? Are young SSCs in the local universe precursors to globular clusters? One characteristic that could link globular clusters to SSCs is the cluster mass function. Globular clusters in the Galaxy have a characteristic mass of a few $\times 10^{5} \mathrm{M}_{\odot}$, reflecting what appears to be a near-universal globular cluster mass function (GCMF) (Harris, 1998). Were globular clusters born with this mass function? Or is the present globular cluster mass function the result of evolution due to dynamical forces such as tidal stripping and disk shocking over billions of years (de Grijs, 2007; Fall and Rees, 1977, Gnedin and Ostriker, 1997, McLaughlin and Fall |2008, Parmentier and Gilmore 2007)? SSCs, as potential precursors to globular clusters, could give us valuable information about the nature vs. nurture question for the GCMF. Is the initial cluster mass function (ICMF) for SSCs universal, and if so, what is it? How does it evolve?

The ICMF has been extensively studied in the Antennae galaxies, where large numbers of SSCs facilitate the statistics. The Antennae are a nearby (13.3 Mpc, Saviane et al. 2008) IR-bright pair of gas-rich interacting galaxies. HST images revealed a system of thousands of young SSCs spread across the galaxy pair (Whitmore and Schweizer, 1995; Whitmore et al., 1999). ISO imaging determined that the youngest clusters and brightest infrared emission occur in a dusty and embedded region between the two galaxies (Mirabel et al., 1998). Cluster masses and ages for the visible SSCs of the Antennae have been obtained from multicolor (UBVRI and $\mathrm{H} \alpha$ ) HST photometry combined with modeling of the cluster colors and luminosities with STARBURST99 (Leitherer et al., 1999, Vázquez and Leitherer, 2005) The cluster mass function (CMF) in the Antennae is power law with $\alpha \sim-2$ over the range of masses $10^{4}-10^{6} \mathrm{M}_{\odot}($ Fall et al. 2005). The median cluster mass in the Antennae appears to be about an order of magnitude less than the median globular cluster mass of giant ellipticals such as M87 (Harris, 2002). Ages determined from the SSC colors in the Antennae range from $10^{6}-10^{8}$ yr (Zhang and Fall, 1999), with a median of 10-20 Myr (Fall et al., 2005; Mengel et al., 2005), but with a small population extending to more than $10^{9} \mathrm{yrs}$. If the SSC system of the Antennae is typical, one could infer that the characteristic mass of globular clusters is due to selective evolution of clusters of different mass (Fall and Zhang, 2001). However, the fact that $70 \%$ of the clusters in the Antennae are less than $\sim 10-20$ Myr of age could indicate that very few if any of the Antennae clusters will survive to become globular clusters. 
Cluster mass functions have been determined for a number of other nearby galaxies, mostly inferred from cluster luminosity functions. A list of nearby starburst systems is given in de Grijs et al. 2003; many cluster systems appear to have power law slopes of $\alpha \sim-2$.

Direct detection of cluster masses via their stellar velocity dispersions and cluster sizes can be done for the closest systems, thereby giving direct determinations of cluster mass functions. Even in smaller SSC systems, dynamical estimates of cluster masses are an important check on mass functions obtained from cluster luminosity functions. Masses at the high ends are consistent with globular cluster masses. In M82, McCrady \& Graham (2007) find a power law mass distribution with slope of $\alpha=-1.91$ for fifteen SSCs, nearly identical to the Antennae.

An intriguing indication of ICMF evolution is the cluster system in NGC 5253. There are several hundred clusters in this galaxy (Caldwell and Phillips, 1989), many of intermediate age, $\sim 1$ Gyr. There is also a younger population of visible and embedded SSCs with ages of $\sim 2-50 \mathrm{Myr}$ (Alonso-Herrero et al., 2002, Calzetti et al. 1997; Gorjian et al., 2001). The cluster mass function for the intermediate age clusters appears to turn over at a mass of $5 \times 10^{4} \mathrm{M}_{\odot}$, while the younger SSCs have a power law mass function (Cresci et al. 2005). Parmentier et al. (2008) suggest that the evolution of the ICMF is due to star formation efficiency (see Section 7).

Future work on stellar cluster mass functions in the coming decade will be done from space with HST and JWST and from the ground with IR AO observations. High resolution and sensitivity are important for these studies, since fields where SSCs are found are usually very crowded. Infrared observations are critical for getting the mass functions of the youngest clusters, the best reflection of the ICMF, since they are likely to be embedded in regions of high extinction.

\section{What environments lead to extreme star formation?}

In Figure 2 is shown the nearby M81-M82-NGC 3077 interacting group of galaxies (Yun et al., 1994). On the left is the Palomar Sky survey image, showing the stellar luminosity; on the right, is the VLA mosaic of the group in the $21 \mathrm{~cm}$ line of HI. The ties that bind this group are obvious in the $21 \mathrm{~cm}$ line emission. M82 is one of the best-known starburst galaxies, with $L_{I R} \sim 6 \times 10^{10} \mathrm{~L}_{\odot}$, and an estimated $10^{4}-10^{5} \mathrm{O}$ stars $\left(\mathrm{N}_{\mathrm{Lyc}} \sim 8 \times 10^{54} \mathrm{~s}^{-1}\right)$. NGC 3077 also has a modest starburst, of $\mathrm{L}_{\mathrm{IR}} \sim 3 \times 10^{8} \mathrm{~L}_{\odot}\left(\mathrm{N}_{\mathrm{Lyc}} \sim 2 \times 10^{52} \mathrm{~s}^{-1}\right.$ : Sanders et al. 2003$)$, and M81 has a mildly active nucleus. Clearly the conditions for "extreme" star formation are favorable in this group. The atomic hydrogen of this interacting group has its own history, which is different from that of the stars within the galaxies; there is evidence that some of the starburst activity is caused by a delayed "raining down" of orbiting gas onto the galaxies several Myr after their closest encounters (Meier et al., 2001).

The most luminous infrared galaxies in the universe, with $\mathrm{L}_{\mathrm{IR}}>10^{11} \mathrm{~L}_{\odot}$, are merging and interacting systems, and these tend to be systems dominated by star formation. Our knowledge of the stellar distributions is greater than our knowledge 
of the gas: potentially many groups of galaxies have the connected appearance of the M81 group with tidal loops in atomic hydrogen, and perhaps even in molecular gas, since starburst galaxies are especially rich in molecular gas (Mirabel and Sanders, 1989). Arrays with wide-field capability, such as the Allen Telescope Array, the Green Bank Telescope, and the SKA, are well-suited to the mapping of large HI fields in nearby galaxy groups.

Star formation efficiency (SFE) is an important characteristic of the star formation process, since it is a measure of how efficiently molecular clouds are turned into stars. SFE is closely tied to the "infant mortality" of SSCs described in the next section. There are numerous measures of the efficiency of star formation, broadly defined as the proportion of star formation per unit gas. The Schmidt law, in which stars follow a power law correlation with density (Schmidt, 1959), or the corresponding Kennicutt law, in terms of gas surface density (Kennicutt, 1998|b) show that star formation on global scales in galaxies is correlated with gas density. The observable $\mathrm{L}_{\mathrm{IR}} / \mathrm{M}\left(\mathrm{H}_{2}\right)$ is also used as an indication of SFE.

While from a global perspective the total gas content, $\mathrm{HI}+\mathrm{H}_{2}$, of galaxies appears to be well correlated with star formation tracers (Crosthwaite and Turner, 2007, Wong and Blitz, 2002), observations of star-forming regions in the Galaxy indicate that stars form from molecular gas clouds, rather than atomic. CO observations show a good correlation of $\mathrm{L}_{\mathrm{CO}}$ with $\mathrm{L}_{\mathrm{IR}}$ (Sanders et al., 1986; Young et al., 1996; Young and Scoville, 1991, Young et al., 1986).

The atomic and molecular gas distribution in the spiral galaxy M83 is shown in Figure 3 along with an overlay of the GALEX ultraviolet image. This figure illustrates the general result that while the atomic hydrogen disk can far exceed the visible disk of a spiral galaxy, the optical portion of a spiral galaxy is primarily molecular gas. Gas that forms stars is molecular. While a good correlation of star formation tracers is seen with $\mathrm{CO}$ emission, an even tighter correlation is seen be-

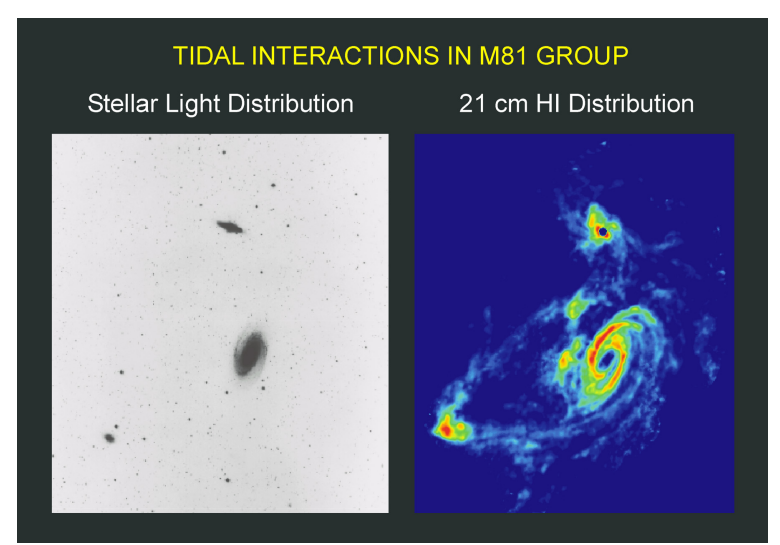

Fig. 2 The M81-M82-NGC 3077 group. (Left) Palomar Sky Survey Image. (Right) VLA image of the $21 \mathrm{~cm}$ line of HI. Yun et al. (1994). Credit: NRAO/AUI/NSF. 

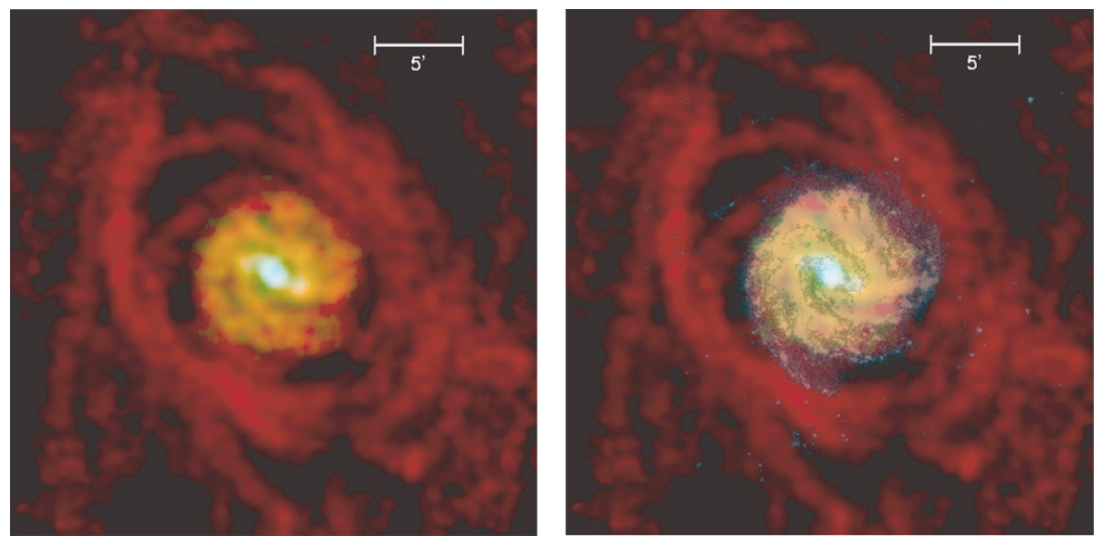

Fig. 3 (Left) The barred spiral galaxy M83. Red is a VLA image of HI $21 \mathrm{~cm}$ line emission, Tilanus and Allen (1993), and yellow is CO emission mapped with the NRAO 12 Meter Telescope, Crosthwaite et al. (2002). (Right) Neutral gas with GALEX image overlay; GALEX image, Thilker et al. (2005), Gil de Paz et al. (2007).

tween star formation and the dense $\left(\mathrm{n}>10^{5-6} \mathrm{~cm}^{-3}\right)$ gas tracer HCN (Gao and Solomon, 2004). This should not be surprising, since denser gas is more likely to form stars.

What complicates the study of molecular gas in star-forming regions is the necessity of using proxies, generally $\mathrm{CO}$, to map out the distribution of $\mathrm{H}_{2}$. A conversion factor between $\mathrm{CO}$ line intensity and $\mathrm{H}_{2}$ mass seems to work well in the Galaxy, but will it do as well in ESF environments?

Because of high energies of its first excited states, $\mathrm{H}_{2}$ tends to be in the ground state for temperatures less than 100K. Most Galactic giant molecular clouds (GMCs) have temperatures of 7-12K (Sanders et al., 1985; Scoville et al., 1987), although clouds in starbursts can be warmer. By contrast, $\mathrm{CO}$ is relatively abundant, easily excited and thermalized, with a lowest energy level equivalent temperature $E / k \sim 5.5 \mathrm{~K}$. For these reasons, $\mathrm{CO}$ lines are generally very optically thick. Yet CO is observed to be a good tracer of mass (Solomon et al. 1987). This is because Galactic disk GMCs appear to be turbulently supported against gravity, and near virial equilibrium (Myers, 1983). GMCs, which consist of optically thick clumps with turbulent motions larger than systematic motions, have line profiles are Gaussian in spite of high optical depths, and "Large Velocity Gradient" (Sobolev approximation) radiative transfer holds (Wolfire et al. 1993). The empiricallydetermined Galactic conversion factor, $\mathrm{X}_{\mathrm{CO}}=\mathrm{N}_{\mathrm{H} 2} / \mathrm{I}_{\mathrm{CO}}$, is thus a dynamical mass tracer (Dickman et al., 1986; Solomon et al. 1987), in effect a Tully-Fisher relation for molecular clouds. Gamma ray observations indicate that a conversion factor of $\mathrm{X}_{\mathrm{CO}}=1.9 \times 10^{20} \mathrm{~cm}^{-2}\left(\mathrm{~K} \mathrm{~km} \mathrm{~s}^{-1}\right)^{-1}$ predicts $\mathrm{H}_{2}$ mass to within a factor of two within the Galaxy, with some radial variation (Strong et al., 1988, 2004). As an indicator of dynamical mass, $X_{C O}$ may actually be more robust than optically thin gas tracers in extreme environments, since mass estimates based on optically thin trac- 
ers depend upon temperature and relative abundance (Dame et al. 2001; Maloney and Black, 1988).

While the CO conversion factor seems to work well in the Galaxy, and as a dynamical mass tracer may be more robust than tracers that are abundance-dependent, the association of $\mathrm{CO}$ and $\mathrm{H}_{2}$ has not been extensively tested in extreme environments. There are clearly some situations in which $X_{C O}$ fails to work well. The Galactic value of $X_{C O}$ does not give good masses for the gas-rich centers of ultraluminous galaxies. In Arp 220, it overestimates the mass by a factor of $\sim 5$ due to a gas-rich nucleus, consisting of two counterrotating disks (Sakamoto et al., 1999), which are dominated by a warm, pervasive molecular gas in which systematic motions dominate over turbulence (Downes and Solomon, 1998; Downes et al., 1993; Solomon et al. 1997). $X_{C O}$ also appears to overpredict $\mathrm{H}_{2}$ masses in the centers of local gasrich spiral galaxies, including our own (Dahmen et al. 1998) by factors of 3-4. $\mathrm{CO}$ appears to systematically misrepresent $\mathrm{H}_{2}$ mass in spiral galaxies when the internal cloud dynamics may be different from Galactic disk clouds, such as in the nuclear regions where tidal shear visibly elongates clouds, causing systematic cloud motions to dominate (Meier and Turner, 2004; Meier et al., 2008). It may also fail where cloud structure may fundamentally differ from Galactic clouds, as in the LMC (Israel et al. 1986), where magnetic fields may be dynamically less important (Bot) et al. 2007).

For an understanding of the links between star formation and molecular gas in ESF regions we require improved confidence in molecular gas masses in environments that are atypical of the Galaxy. Systematic studies of molecular clouds in different tracers of molecular gas, including dust, in ESF galaxies at high resolution in the millimeter and submillimeter with ALMA, CARMA, Plateau de Bure, and SMA will shed light on when we can confidently use $\mathrm{CO}$ as a tracer of molecular gas mass, and under what conditions it ceases to be a good tracer.

Environmental factors other than gas mass are also important in the fostering of star formation, but these are not as yet well understood. Tacconi and Young (1990) concluded that the efficiency of massive star formation is higher in spiral arms than between the arms, consistent with the "strings of pearls along the spiral arms" description of nebulae by Baade (1957). Clearly star formation is enhanced by spiral arms, but how their large-scale influence trickles down in a turbulent GMC to a parsec-scale core is not at all clear (Padoan et al., 2007). Starburst rings also appear to facilitate star formation, particularly young SSCs (Barth et al. 1995: Maoz et al. 2001, 1996). SFE appears to steadily increase with the ferocity of the star formation. While $\mathrm{L}_{\mathrm{IR}} / \mathrm{M}_{\mathrm{H}_{2}} \sim 4 \mathrm{~L}_{\odot} / \mathrm{M}_{\odot}$ for the Galaxy, it is $\sim 10-20 \mathrm{~L} \odot \mathrm{M}_{\odot}$ in regions of active star formation, 20-100 in ULIRGs (Sanders et al., 1986). These studies rely on $L_{I R}$, which may have contributions from older stellar populations. Studies of star formation efficiency using tracers of recent star formation are ongoing.

Extreme star formation should require extreme amounts of molecular gas, and luminous infrared galaxies have plenty of it (Downes et al., 1993, Sanders and Mirabel, 1985, 1996, Young et al., 1986). The Antennae interacting system, with its thousands of young SSCs, contains exceptional amounts of gas. A CO image made with the Owens Valley Millimeter Array by Wilson et al. (2000, 2003) 


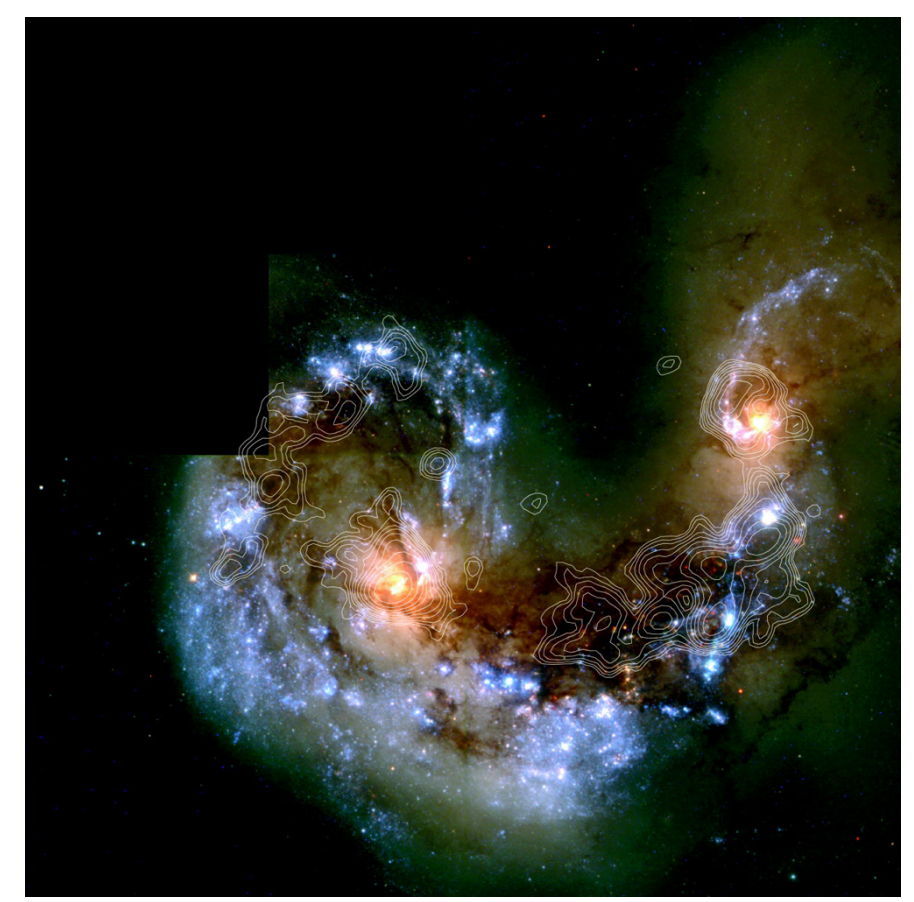

Fig. $4 \mathrm{CO}$ in the Antennae. Contours are emission in the $\mathrm{J}=1-0$ line of $\mathrm{CO}$ at $3 \mathrm{~mm}$, imaged with the Owens Valley Millimeter Array. In color is the HST image. Wilson et al. (2000), Whitmore et al. (1999). Credit: C. Wilson.

is shown on the HST image of (Whitmore et al., 1999) in Figure 4 , The greatest concentration of molecular gas is in the dusty, obscured region between the two galaxies where the brightest infrared emission is found (Mirabel et al., 1998). There is an estimated $10^{9} \mathrm{M}_{\odot}$ of molecular gas in the Antennae, supporting a current star formation rate of $\mathrm{N}_{\mathrm{Lyc}} \sim 10^{54} \mathrm{~s}^{-1}$ (Stanford et al. 1990, for $\mathrm{D}=13 \mathrm{Mpc}$, and $X_{C O} \sim 2 \times 10^{20} \mathrm{~cm}^{-2} \mathrm{~K} \mathrm{~km} \mathrm{~s}^{-1}$ ). Based on the total mass of K-band selected clusters, the SFE is about 3-6\% (Mengel et al., 2005), slightly higher than Galactic efficiencies on these scales (Lada et al., 1984) but not by much. $\mathrm{H}_{2}$ emission observed using Spitzer in the dusty collision region suggests that "pre-starburst" shocks may trigger the star formation there (Haas et al. 2005) as seen in other interacting ULIRGS (Higdon et al., 2006). This is a good illustration of the "Super Giant Molecular Clouds" posited for interacting systems by Harris (2002); Harris and Pudritz (1994). Like the stellar cluster mass function, which is power law with $\alpha \sim-2$, the mass function of giant molecular clouds in the Antennae is also power law, but with a slightly different slope, $\alpha \sim-1.4$ (Wilson et al., 2003).

A counterexample to the "lots of gas, lots of stars" theory is the case of NGC 5253. In this starburst dwarf galaxy, the star formation efficiency appears to be extremely high, SFE $\sim 75 \%$ on $100 \mathrm{pc}$ scales, with $\mathrm{H}_{2}$ masses based on both $\mathrm{CO}$ (Meier et 
al. 2002, Turner et al., 1997) and dust emission (Turner et al. 2008, in prep.) This SFE is nearly two orders of magnitude higher than generally seen on GMC spatial scales in the Galaxy. NGC 5253 has several hundred young clusters, including several SSCs (Calzetti et al., 1997; Gorjian, 1996). Why is this galaxy so parsimonious in its usage of gas compared to the Antennae? One difference between this starburst and that of the Antennae is that NGC 5253 is a dwarf galaxy, with an estimated mass of $\sim 10^{9} \mathrm{M}_{\odot}$, which was probably originally a gas-poor dwarf spheroidal galaxy that has accreted some gas (Caldwell and Phillips, 1989). Unlike the Antennae, which are in the midst of a full-blown major merger, NGC 5253 is relatively isolated, although part of the Cen A-M83 group (Karachentsev et al., 2007). The prominent dust lane entering the minor axis is the probable cause of the starburst. Molecular gas is present in the dust lane, and this gas is observed to be falling into the galaxy near the current starburst (Kobulnicky and Skillman, 2008, Meier et al. 2002). Radio recombination line emission imaged at high resolution with the VLA of the central "supernebula" shows a velocity gradient in the same direction as that of the infalling streamer (Rodríguez-Rico et al., 2007). High star efficiency is a necessary condition that the SSCs can evolve into globular clusters (Goodwin, 1997). NGC 5253 may be the best case yet for a galaxy in which the clusters might survive to become globular clusters.

The high resolution and sensitivity of ALMA, the new CARMA array, Plateau de Bure and SMA will allow many more such starburst systems to be imaged in molecular lines for study of the variation in SFE with environment. Star formation efficiency is a key parameter in the formation of long-lived clusters.

\section{Super Star Cluster Mortality}

Birth and death go hand in hand in young SSCs, since O stars barely stop accreting before they die (Zinnecker and Yorke, 2007). During their short lifetimes, O stars find many different ways to lose mass. Windy and explosive by nature, $\mathrm{O}$ stars emit copious numbers of destructive ultraviolet photons and are responsible for much of the mass loss and mechanical feedback within a star cluster. Outflows, wind bubbles, LBV mass ejections, and SNR from a single $O$ star can influence a region the size of a young SSC; imagine what an SSC consisting of thousands of O stars can do to a parsec-scale volume! Figure 5 illustrates a few of the many ways that $\mathrm{O}$ stars can destructively interact with their environments: $\mathrm{CO}$ outflows in adolescence (1 Myr); LBV outflows similar to that responsible for the Homunculus Nebula in early adulthood (2-3 Myr); Wolf-Rayet wind bubbles at retirement (3-4 Myr); death by supernova (5-10 Myr). If the young cluster manages to survive through the paroxysms of its riotous $\mathrm{O}$ star siblings, then there is most likely a molecular cloud nearby to unbind it.

There is strong evidence that most SSCs in the local universe will not survive to old age. The odds of survival even in the relatively benign environments of the Galactic disk and halo are slim. Only an estimated $\sim 7 \%$ of embedded young clusters 
in the solar neighborhood will live to the age of the Pleiades (Lada and Lada, 2003). Dynamical models of the Galactic globular cluster system suggest that as much as $75 \%$ of the Galactic stellar halo may have originally been in the form of globular clusters (Gnedin and Ostriker, 1997, Shin et al. 2008), which now account for only $1 \%$ of visible halo stars (Harris, 1998).

The first hurdle that a young SSC must overcome is star formation efficiency, defined here as $\eta=M_{\text {stars }} /\left(M_{\text {stars }}+M_{\text {gas }}\right)$. The gas mass contribution includes contributions from ionized gas and atomic and molecular gas from the natal clouds. Nominally $\eta>50 \%$ is required to leave a bound cluster (Hills, 1980, Lada et al., 1984: Mathieu, 1983). Lower values of $\eta \sim 30 \%$ can be accommodated if the cluster loses stars, but retains a smaller bound core, leading to smaller clusters. The cluster may also survive if gas is lost quasistatically, so that the cluster adjusts to the new equilibrium (Kroupa and Boily, 2002); it may also survive, although with a significantly reduced stellar mass, if it suffers rapid mass loss ("infant weight-loss") early on (Bastian and Goodwin 2006). Models suggest that clusters with masses less than $10^{5} \mathrm{M}_{\odot}$ lose their residual gas quickly, and that $95 \%$ of all clusters are so destroyed within a few tens of Myr, and that rapid gas expulsion may give a natural explanation for the lognormal PDMF for globular clusters (Baumgardt et al. 2008).

Based on the SFE/ $\eta$ as observed in the Galaxy, the picture looks bleak for young SSCs. On the sizescales of giant molecular clouds, $\eta$ is at most a few percent (Lada and Lada. 2003), a number that appears to be determined by the turbulent dynamics of clouds (Krumholz et al., 2006; Padoan and Nordlund, 2002; Padoan et al. 2007). On smaller scales in the Galaxy, $\eta \sim 10 \%-30 \%$ (NGC 3603; Nürnberger et al. 2002), which is still rather small to preserve a significant bound cluster on globular cluster scales.

There is both fossil and structural observational evidence that clusters dissolve. The vast majority of bright SSCs with masses over $10^{5} \mathrm{M}_{\odot}$ in known SSC systems are less than $10 \mathrm{Myr}$ in age (Bastian et al., 2005; Mengel et al. 2005). From STIS spectroscopy, Tremonti et al. (2001) and Chandar et al. (2005) find that the field stars in the nearby starburst galaxy NGC 5253 can be modeled by dispersed cluster stars, with cluster dissolution timescales of 7-10 Myr. In the Antennae system, Fall et al. (2005) find that the number of clusters falls sharply with age, with $\sim 50 \%$ of the stars in clusters having dispersed after 10 Myrs. They estimate that at least 20\% and perhaps all, of the disk stars in the Antennae have formed within clusters. On the other hand, there is fossil evidence, in the form of globular clusters, that large clusters can and do survive for many Gyr.

Star formation efficiency and cluster disruption may imprint upon cluster mass functions. Parmentier et al. (2008) suggest that at $\eta \sim 20 \%$, a power-law core mass function turns into a bell-shaped cluster mass function, while at higher efficiencies the power law is preserved. Gieles \& Bastian (2008) suggest that the maximum cluster mass and age is a diagnostic of cluster disruption, and they see evidence in cluster mass function, that formation/disruption does vary among galaxies.

The question remains of how globular clusters have managed to live to such a ripe old age. Can we identify SSCs forming today that might indeed live to become 10 billion years old? What initial conditions favor SSC longevity? Going to deeper 

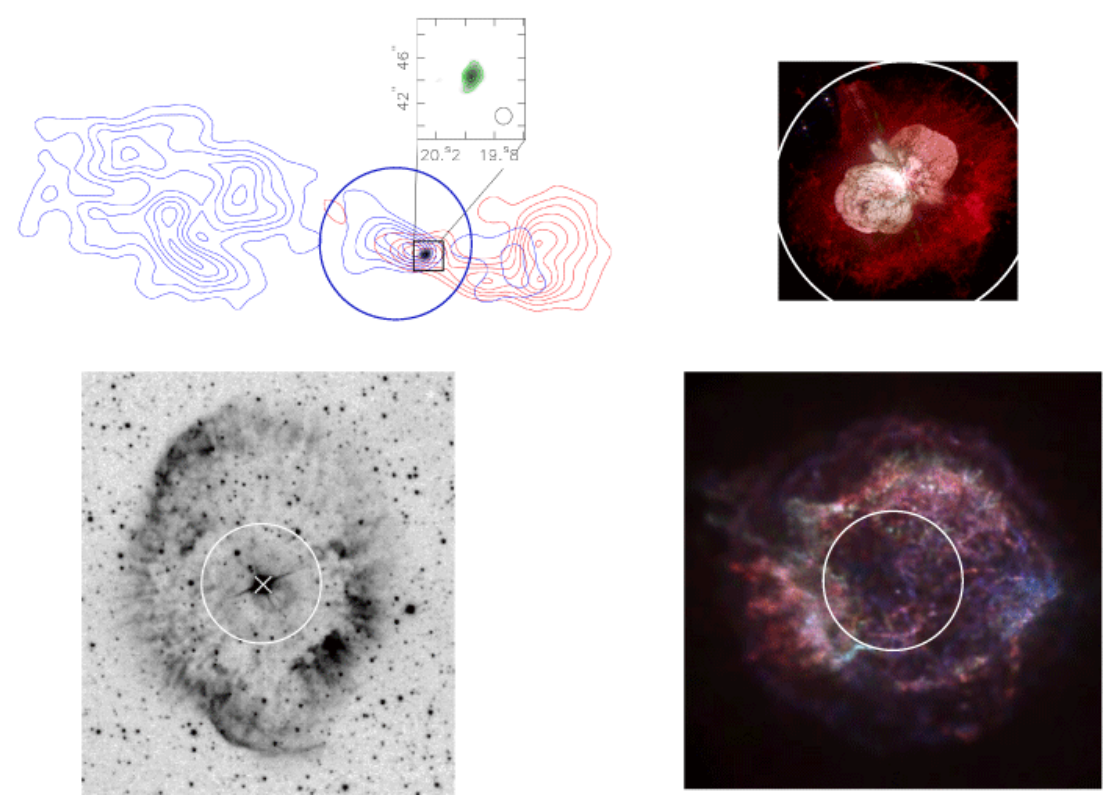

Fig. 5 The many ways that $\mathrm{O}$ stars can be destructive. Circles represent a region $1 \mathrm{pc}$ across, the size of the core of an SSC. (top left) Owens Valley Millimeter Array image of the CO outflow source around the massive protostar G192.16-3.82, Shepherd and Kurtz (1999). (top right) The Homunculus Nebula in Eta Carina imaged by HST, Morse et al. (1998). (lower left) Wolf-Rayet bubble RCW58 in $\mathrm{H} \alpha$, Gruendl et al. (2000). (lower right) Chandra image of the 1000-yr-old SNR Cas A, Hughes et al. (2000).

limits in the cluster luminosity function could illuminate the connection between today's SSCs and the older globular cluster population (Chandar et al., 2004b). This is an extremely active area of research, but there are currently few examples of high resolution studies of the efficiency of star formation on GMC sizescales in starbursts. ALMA will have the sensitivity and resolution to enable these studies in many nearby galaxies.

\section{Radiative Feedback: Effects on Molecular Clouds and Chemistry}

The starburst galaxy, M82, has one of the earliest known and best studied examples of a galactic wind. Both mechanical luminosity in the form of stellar winds and supernovae and radiative luminosity from starbursts are feedback mechanisms that can potentially disrupt star formation and end the starburst phase. Yet there are galaxies, such as the Antennae, observed to have thousands of SSCs spread over regions of hundreds of pc extent, with cluster ages spanning many tens of Myr during tidal in- 
teractions lasting $\sim 100 \mathrm{Myr}$. Evidently, episodes of intense star formation can take place over periods of time far longer than the lifetimes of individual massive stars in spite of feedback. The subject of galactic winds and feedback is a large and active one, and has been recently reviewed by Veilleux et al. (2005). The effects of feedback on denser molecular gas in ESF regions is not yet well characterized, and it is the molecular gas from which the future generations of stars will form.

Starburst feedback and star formation occur on different spatial scales. Giant molecular clouds consist of clumps that are governed by turbulence; only a small fraction of these clumps contain cores, which are the regions that collapse to form stars or star clusters (McKee and Ostriker. 2007). Current computational models of turbulent clouds can explain the canonical star formation efficiencies of a few percent as that fraction of turbulent clumps that become dense enough for gravity to dominate (Krumholz et al., 2006; Padoan and Nordlund, 2002; Padoan et al., 2007). Once a core begins to collapse, free fall is rapid and there is little time for feedback to operate. It is more likely that feedback operates on longer-lived and lower density molecular cloud envelopes dominated by turbulence, but how this large scale effect communicates down to the small and rapidly collapsing star forming cores is unclear (Elmegreen, 2007, Krumholz and Tan, 2007; Padoan et al., 2007). If rich star clusters form stars for several dynamical times, the energetic input feedback could become important (Tan et al., 2006).

One surprising characteristic of the interstellar medium in regions of ESF is the ubiquity of dense $\left(\mathrm{n}_{\mathrm{e}} \sim 10^{4-5} \mathrm{~cm}^{-3}\right)$, "compact" HII regions, a stage of star formation that should be fleeting and relatively rare. First detected spectroscopically in dwarf galaxies, such as NGC 5253 and He 2-10 (Beck et al. 2000, Kobulnicky and Johnson, 1999; Turner et al. 1998), these nebulae are detected by their high freefree optical depths at $\mathrm{cm}$ wavelengths. These are the ESF analogs of dense Galactic "compact" HII regions, only much larger in size because of the high Lyman continuum rates from these large clusters. If the expansion of HII regions is governed by classical wind bubble theory, then the dynamical ages implied by the sizes of these HII regions are extremely short, tens of thousands of years. M82-A1 is a young SSC with both a visible HII region and a cluster, in which the dynamical age of the HII region is too small for the cluster age (Smith et al. 2006). The HII region around the Galactic cluster NGC 3603 is also too small for the cluster age (Drissen et al. 1995). These may be scaled-up versions of the classic Galactic ultracompact HII problem, in which there are "too many" compact HII regions given their inferred dynamical ages (Dreher et al., 1984, Wood and Churchwell, 1989). A possible explanation for the long lifetimes of these HII regions is confinement by the high pressure environment of nearby dense $\left(n_{H_{2}}>10^{5} \mathrm{~cm}^{-3}\right)$ and warm molecular clouds (e.g., de Pree et al. 1995; Dopita et al., 2005, 2006). Radiative cooling may provide an important energy sink for the output of young super star clusters (Silich et al. 2007). However, the standard wind-blown bubble theory that is generally applied to the development of Galactic HII regions (Castor et al. 1975; Chevalier and Clegg, 1985) may not always apply to the HII regions surrounding SSCs, which are massive enough to exert a non-negligible gravitational pull on their HII regions (Kroupa and Boily, 2002). The "supernebula" in NGC 5253 appears to be gravity-bound, if not in equilibrium 


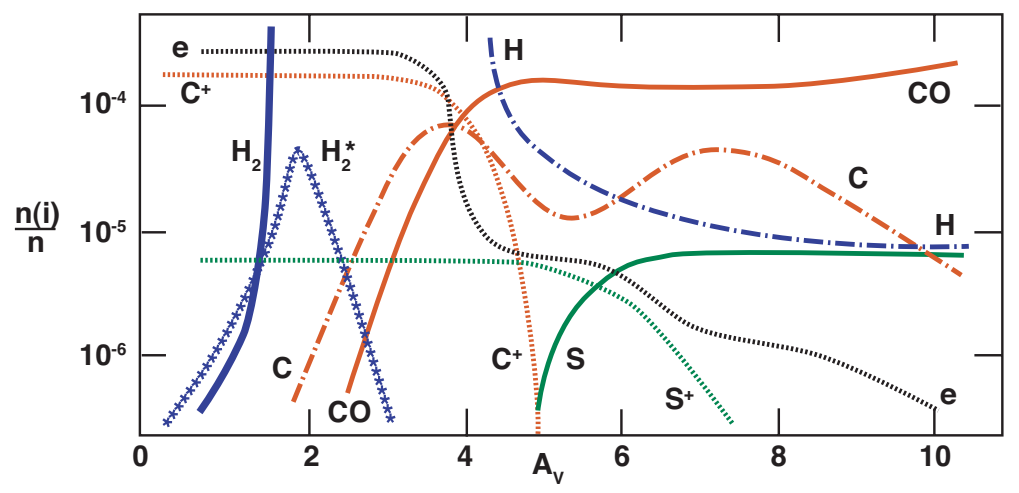

Fig. 6 Schematic of the ionization structure of the Orion photodissociation region (PDR), with relative elemental abundance plotted versus visual extinction. Adapted from Tielens \& Hollenbach (1985) and Tielens (2005).

(Turner et al. 2003). Gravity could either stall the expansion of an SSC HII region's expansion or create a cluster wind akin to a stellar wind, depending on conditions. It is clear that the high interstellar pressures in starburst regions are critical to their development, and to the evolution of the nearby molecular clouds.

Molecular gas has a tremendous ability to absorb energy and radiate it away. This could explain the ability of galaxies to sustain starburst events of extended duration such as the one that has produced the thousands of SSCs in the Antennae. Irradiation causes heating, ionization, dissociation, and pronounced chemical changes in molecular clouds. It also provides us with a rich spectrum of potential diagnostics of radiative feedback.

In Figure 6 is shown a schematic of the ionization structure of a photodissociation region (PDR), adapted from Tielens and Hollenbach (1985). (For a full description of PDRs, see Tielens 2005 or Hollenbach and Tielens 1999 and also Bertoldi and Draine 1996; Draine and Bertoldi 1996; Kaufman et al. 2006, 1999, Tielens and Hollenbach 1985.) Molecular clouds tend to form $\mathrm{H}_{2}$ at $\mathrm{A}_{\mathrm{V}}<\sim 1$. The translucent edge to the molecular cloud can be quite warm, a few hundred $\mathrm{K}$, warm enough for excited $\mathrm{H}_{2}$ to be visible. Between $A_{V}=1$ and $A_{V} \sim 5$, while the clouds are molecular, they also have significant abundances of heavy ions such as $\mathrm{C}^{+}$and $\mathrm{S}^{+}$. The presence of ions can drive a rich gas-phase chemistry through ion-molecule reactions. The high temperatures can also liberate molecules that have formed on the surfaces of grains in the form of ices in the coldest clouds. Warming the grains in either shocks or radiatively in PDRs brings these molecules into the gas phase. PAH emission is also bright from these regions, and dominates Spitzer images in the $8 \mu \mathrm{m}$ IRAC band where it shows a close association with star-forming regions (Galliano et al. 2008: Peeters et al., 2004). These chemical diagnostics have been used to great effect in modeling the effects of protostars on their surrounding protostellar disks, and in determining the shapes and orientations of disks (van Dishoeck and Blake, 1998). Clouds near sources of high X-ray radiation are subject to a similar phe- 
nomenon, but with chemistry that is driven by a hard radiation field; these regions are called "XDRs" (Lepp and Dalgarno, 1996; Maloney et al., 1996; Meijerink and Spaans, 2005, Meijerink et al., 2006).

It might seem that processes that occur on scales of $A_{v} \sim 1-5$ would be difficult to detect in other galaxies, on the spatial scales of GMCs, but this is not the case. Molecular clouds are porous, and there are many surfaces within clouds of relatively low $A_{v}$ individually; an estimated $90 \%$ of molecular gas is in PDRs (Hollenbach and Tielens, 1999). Some of the first indications of the importance of PDR chemistry were the detections of warm $\mathrm{CO}$ and the tracers of warmed, potentially shocked, gas such as the CII $158 \mu \mathrm{m}$ line in starburst galaxies (Mauersberger and Henkel, 1991; Stacey et al., 1991). Temperatures as high as 400-900K have been inferred from lines of interstellar ammonia (Mauersberger et al., 2003).

Lines of numerous heavy molecules have been detected from other galaxies, and the brightest sources are the star-forming galaxies. Molecules such as formaldehyde $\left(\mathrm{H}_{2} \mathrm{CO}\right)$, methanol $\left(\mathrm{CH}_{3} \mathrm{OH}\right)$, and cyanoacetylene $\left(\mathrm{HC}_{3} \mathrm{~N}\right)$ have been detected in nearby starburst galaxies (Mangum et al., 2008; Martín et al. 2006; Meier and Turner. 2008). Many of these models can provide discriminants between PDR and XDR-heated gas (Aalto et al., 2007, Meijerink et al., 2006). A spectral line survey of NGC 253 in the $2 \mathrm{~mm}$ atmospheric window reveals 111 identifiable spectral features from 25 different molecular species; the spectrum suggests that the molecular abundances in NGC 253 are similar to those of the Galactic Center, with a chemistry dominated by low-velocity shocks (Martín et al. 2006, 2008).

Imaging adds another dimension to the molecular line spectra. In Figure 7 are shown interferometric images in several molecules of the nuclear "minispiral" of the nearby Scd galaxy, IC 342. The lines shown are all at $\lambda=3 \mathrm{~mm}$, and have comparable excitation energies and similar critical densities. The images show a clear variation in cloud chemistry and molecular abundances with galactic location. A principal component analysis (Meier and Turner, 2005) shows that the molecules $\mathrm{N}_{2} \mathrm{H}^{+}$, $\mathrm{HNC}$, and $\mathrm{HCN}$ have similar spatial distributions to $\mathrm{CO}$ and its isotopologues, and are good tracers of the overall molecular cloud distribution. The molecule $\mathrm{CH}_{3} \mathrm{OH}$ (methanol) is well known from Galactic studies to be a "grain-chemistry" molecule, which forms on grain surfaces and is liberated by shocks or warm cloud conditions; here, methanol and HNCO follow the arms of the minispiral. Methanol and HNCO appear to be tracing the gentle shocks of the gas passing through the spiral arms. The final group of molecules, $\mathrm{C}^{34} \mathrm{~S}$ and $\mathrm{C}_{2} \mathrm{H}$ are found in the immediate vicinity $(50 \mathrm{pc})$ of the nuclear cluster; these molecules presumably reflect the intense radiation fields of the nuclear star cluster.

The next decade will see a blossoming of molecular line studies of ESF. The current state of molecular line work in galaxies has been recently reviewed by Omont (2007), and also is represented by contributions in the volume by Bachiller \& Cernicharo (2008). Spectroscopy of ESF gas in galaxies with the APEX, ASTE, CARMA, IRAM 30 m, Plateau de Bure, CARMA, SMA, Spitzer, and the VLA telescopes will continue to probe the star-forming ISM in nearby galaxies through the next decade. Herschel and SOFIA will soon provide spectroscopy of the important $158 \mu \mathrm{m}$ line of CII from PDRs in ESF galaxies. ALMA will add tremendous sen- 

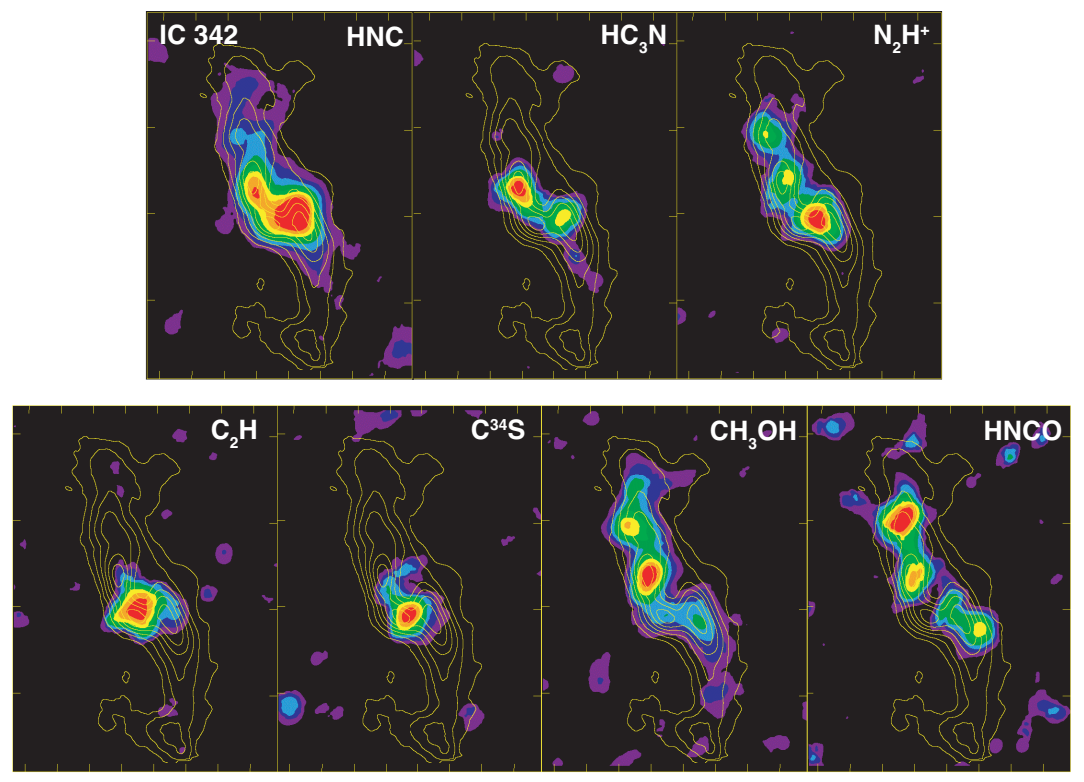

Fig. 7 Spatially-resolved $\left(5^{\prime \prime}=75 \mathrm{pc}\right)$ chemistry of the central $300 \mathrm{pc}$ of the Scd spiral galaxy IC 342. Meier \& Turner (2005). Panels at top show molecules that are overall molecular gas tracers. Bottom, left: $\mathrm{C}_{2} \mathrm{H}$ and $\mathrm{C}^{34} \mathrm{~S}$ trace clouds in high radiation fields (PDRs); right: $\mathrm{CH}_{3} \mathrm{OH}$ (methanol) and HNCO trace grain-chemistry along the arms of the minispiral.

sitivity, milliarcsecond resolution, $\mathrm{km} / \mathrm{s}$ velocity resolution, access to the southern hemisphere, and submillimeter capability, allowing us to study extreme star formation and extreme star-forming gas and its effects on galaxies in exquisite detail.

Acknowledgements I am grateful to Nate McCrady, David S. Meier, and Andrea Stolte for their helpful discussions and comments, and to Xander Tielens for his good humor and patience.

\section{References}

Aalto, S., Spaans, M., Wiedner, M.C., Hüttemeister, S., Astron. Astrophys., 464, 193-200 (2007)

Alonso-Herrero, A., Rieke, G.H., Rieke, M.J., Scoville, N.Z., Astron. J., 124, 166$182(2002)$

Alonso-Herrero, A., Takagi, T., Baker, A.J., Rieke, G.H., Rieke, M.J., Imanishi, M., Scoville, N.Z., Astrophys. J., 612, 222-237 (2004)

Arp, H., Sandage, A., Astron. J., 90, 1163-1171 (1985)

Baade, W., The Observatory, 77, 165-171 (1957) 
Bachiller, R. and Cernicharo, J., Science with the Atacama Large Array, (New York: Springer) (2008)

Barth, A.J., Ho, L.C., Filippenko, A.V., Sargent, W.L., Astron. J., 110, 1009-1018 (1995)

Bastian, N., Goodwin, S.P., Mon. Not. R. Astron. Soc., 369, L9-L13 (2006)

Bastian, N., Gieles, M., Efremov, Y.N., Lamers, H.J.G.L.M., Astron. Astrophys., 443, 79-90 (2005)

Battinelli, P., Capuzzo-Dolcetta, R., Mon. Not. R. Astron. Soc., 249, 76-83 (1991)

Baumgardt, H., Kroupa, P., Parmentier, G., Mon. Not. R. Astron. Soc., 384, 12311241 (2008)

Beck, S.C., Turner, J.L., Ho, P.T.P., Lacy, J.H., Kelly, D.M., Astrophys. J., 457, 610615 (1996)

Beck, S.C., Turner, J.L., Kloosterman, J., Astron. J., 134, 1237-1244 (2007)

Beck, S.C., Turner, J.L., Kovo, O., Astron. J., 120, 244-259 (2000)

Bertoldi, F., Draine, B.T., Astrophys. J., 458, 222-232 (1996)

Billett, O.H., Hunter, D.A., Elmegreen, B.G., Astron. J., 123, 1454-1475 (2002)

Boily, C.M., Lançon, A., Deiters, S., Heggie, D.C., Astrophys. J., 620, L27-L30 (2005)

Bonnell, I.A., Bate, M.R., Zinnecker, H., Mon. Not. R. Astron. Soc., 298, 93-102 (1998)

Bot, C., Boulanger, F., Rubio, M., Rantakyro, F., Astron. Astrophys., 471, 103-112 (2007)

Brandner, W., Clark, J.S., Stolte, A., Waters, R., Negueruela, I., Goodwin, S.P., Astron. Astrophys., 478, 137-149 (2008)

Burbidge, E.M., Burbidge, G.R., Astrophys. J., 135, 694-710 (1962)

Burbidge, E.M., Burbidge, G.R., Rubin, V.C., Astron. J., 69, 535-535 (1964)

Caldwell, N., Phillips, M.M., Astrophys. J., 338, 789-803 (1989)

Calzetti, D., Meurer, G.R., Bohlin, R.C., Garnett, D.R., Kinney, A.L., Leitherer, C., Storchi-Bergmann, T., Astron. J., 114, 1834-1849 (1997)

Castor, J., McCray, R., Weaver, R., Astrophys. J., 200, L107-L110 (1975)

Chandar, R., Leitherer, C., Tremonti, C., Calzetti, D., Astrophys. J., 586, 939-958 (2003)

Chandar, R., Leitherer, C., Tremonti, C.A. 2004, Astrophys. J., 604, 153-166 (2004)

Chandar, R., Leitherer, C., Tremonti, C.A., Calzetti, D., Aloisi, A., Meurer, G.R., de Mello, D., Astrophys. J., 628, 210-230 (2005)

Chandar, R., Whitmore, B., Lee, M.G., Astrophys. J., 611, 220-244 (2004)

Chevalier, R.A., Clegg, A.W., Nature, 317, 44-45 (1985)

Clark, J.S., Fender, R.P., Waters, L.B.F.M., Dougherty, S.M., Koornneef, J., Steele, I.A., van Blokland, A., Mon. Not. R. Astron. Soc., 299, L43-L47 (1998)

Clark, J.S., Negueruela, I., Crowther, P.A., Goodwin, S.P., Astron. Astrophys., 434, 949-969 (2005)

Clarke, C.J., Bonnell, I. A., Mon. Not. R. Astron. Soc., in press (2008)

Condon, J.J., Annu. Rev. Astron. Astrophys., 30, 575 (1992)

Conti, P.S. 1991, Astrophys. J., 377, 115-125

Conti, P.S., Blum, R.D., Astrophys. J., 564, 827-833 (2002) 
Cresci, G., Vanzi, L., Sauvage, M., Astron. Astrophys., 433, 447-454 (2005)

Crosthwaite, L.P., Turner, J.L., Buchholz, L., Ho, P.T.P., and Martin, R.N., Astron. J., 123, 1892-1912 (2002)

Crosthwaite, L.P., Turner, J.L., Astron. J., 134, 1827-1842 (2007)

Crowther, P.A., Beck, S.C., Willis, A.J., Conti, P.S., Morris, P.W., and Sutherland, R.S., Mon. Not. R. Astron. Soc., 304, 654-668 (1999)

Crowther, P.A., Hadfield, L.J., Clark, J.S., Negueruela, I., Vacca, W.D., Mon. Not. R. Astron. Soc., 372, 1407-1424 (2006)

Dahmen, G., Huttemeister, S., Wilson, T.L., Mauersberger, R. 1998, Astron. Astrophys., 331, 959-976

Dale, D.A., et al., Astrophys. J., 646, 161-173 (2006)

Dame, T.M., Hartmann, D., Thaddeus, P. 2001, Astrophys. J., 547, 792-813

de Grijs, R. to appear in "Young massive star clusters-Initial conditions and environments", E. Perez, R. de Grijs, R. M. Gonzalez Delgado, eds., (Springer: Dordrecht) e-prints, 711, arXiv:0711.3540 (2007)

de Grijs, R., ers, P., Bastian, N., Lynds, R., Lamers, H.J.G.L.M., O’Neil, E.J., Mon. Not. R. Astron. Soc., 343, 1285-1300 (2003)

de Jong, T., Clegg, P.E., Rowan-Robinson, M., Soifer, B.T., Habing, H.J., Houck, J.R., Aumann, H.H., Raimond, E., Astrophys. J., 278, L67-L70 (1984)

de Marchi, G., Nota, A., Leitherer, C., Ragazzoni, R., Barbieri, C., Astrophys. J., 419, 658-669 (1993)

de Marchi, G., Clampin, M., Greggio, L., Leitherer, C., Nota, A., Tosi, M., Astrophys. J., 479, L27-L30 (1997)

de Pree, C.G., Nysewander, M.C., Goss, W.M., Astron. J., 117, 2902-2918 (1999)

de Pree, C.G., Rodriguez, L.F., and Goss, W.M., Revista Mexicana de Astronomia y Astrofisica, 31, 39-44 (1995)

Dickman, R.L., Snell, R.L., Schloerb, F.P., Astrophys. J., 309, 326-330 (1986)

Dopita, M.A., et al., Astrophys. J., 619, 755-778 (2005)

Dopita, M.A., et al., ApJS, 167, 177-200 (2006)

Dowell, C.D., Astrophys. J., 487, 237-247 (1997)

Downes, D., Solomon, P.M., Astrophys. J., 507, 615-654 (1998)

Downes, D., Solomon, P.M., Radford, S.J.E., Astrophys. J., 414, L13-L16 (1993)

Draine, B.T., Bertoldi, F., Astrophys. J., 468, 269-289 (1996)

Dreher, J.W., Johnston, K.J., Welch, W.J., Walker, R.C., Astrophys. J., 283, 632-639 (1984)

Drissen, L., Moffat, A.F.J., Walborn, N.R., Shara, M.M., Astron. J., 110, 2235-2241 (1995)

Eisenhauer, F., Quirrenbach, A., Zinnecker, H., Genzel, R., Astrophys. J., 498, 278292 (1998)

Elmegreen, B.G., Astrophys. J., 668, 1064-1082 (2007)

Elmegreen, B.G., ArXiv e-prints, 803, arXiv:0803.3154 (2008)

Fall, S.M., Rees, M.J., Mon. Not. R. Astron. Soc., 181, 37P-42P (1977)

Fall, S.M., Zhang, Q., Astrophys. J., 561, 751-765 (2001)

Fall, S.M., Chandar, R., Whitmore, B.C., Astrophys. J., 631, L133-L136 (2005)

Figer, D.F., Astronomische Nachrichten Supplement, 324, 255-261 (2003) 
Figer, D.F., The Formation and Evolution of Massive Young Star Clusters, 322, 49 (2004)

Figer, D.F., Nature, 434, 192-194 (2005)

Figer, D.F., ArXiv e-prints, 803, arXiv:0803.1619 (2008)

Figer, D.F., in Massive Stars as Cosmic Engines, IAU Symposium, 250, 247-256 (2008)

Figer, D.F., Kim, S.S., Morris, M., Serabyn, E., Rich, R.M., and McLean, I.S., Astrophys. J., 525, 750-758 (1999)

Figer, D.F., Najarro, F., Morris, M., McLean, I.S., Geballe, T.R., Ghez, A.M., Langer, N., Astrophys. J., 506, 384-404 (1998)

Förster Schreiber, N.M., Genzel, R., Lutz, D., Kunze, D., Sternberg, A., Astrophys. J., 552, 544-571 (2001)

Galliano, F., Madden, S.C., Tielens, A.G.G.M., Peeters, E., and Jones, A.P., Astrophys. J., 679, 310-345 (2008)

Gao, Y., Solomon, P.M., Astrophys. J., 606, 271-290 (2004)

Gascoigne, S.C.B., Kron, G.E., Publ. Astron. Soc. Pac., 64, 196-200 (1952)

Gaume, R.A., Claussen, M.J., de Pree, C.G., Goss, W.M., Mehringer, D.M., Astrophys. J., 449, 663-673 (1995)

Gehrz, R.D., Sramek, R.A., Weedman, D.W., Astrophys. J., 267, 551-562 (1983)

Genzel, R., Cesarsky, C.J. 2000, Annu. Rev. Astron. Astrophys., 38, 761-814

Genzel, R., et al., Astrophys. J., 498, 579-605 (1998)

Gieles, M., Bastian, N., Astron. Astrophys., 482, 165-171 (2008)

Gil de Paz, A., et al., ApJS, 173, 185-255 (2007)

Gilbert, A.M., Ph.D.Dissertation, University of California, Berkeley (2002)

Gilbert, A.M., et al., Astrophys. J., 533, L57-L60 (2000)

Goodwin, S.P. 1997, Mon. Not. R. Astron. Soc., 286, 669-680 (1997)

Gorjian, V., Astron. J., 112, 1886-1893 (1996)

Gorjian, V., Turner, J.L., Beck, S.C., Astrophys. J., 554, L29-L33 (2001)

Gnedin, O.Y., Ostriker, J.P., Astrophys. J., 474, 223-255 (1997)

Gruendl, R.A., Chu, Y.-H., Dunne, B.C., Points, S.D., Astron. J., 120, 2670-2678 (2000)

Haas, M., Chini, R., Klaas, U., Astron. Astrophys., 433, L17-L20 (2005)

Hadfield, L.J., Crowther, P.A., Mon. Not. R. Astron. Soc., 368, 1822-1832 (2006)

Harayama, Y., Eisenhauer, F., Martins, F., Astrophys. J., 675, 1319-1342 (2008)

Harris, W.E., Extragalactic Star Clusters, 207, 545 (2002)

Harris, W.E., Astron. J., 112, 1487-1488 (1996)

Harris, W.E., in Galactic Halos, ed. D. Zaritsky, (San Francisco:A. S. P.) 136, 33-41 (1998)

Harris, W.E., Harris, H.C., in Astrophysical Quantities, ed. A. N. Cox (Springer, London), 545-568 (2000)

Harris, W.E., Pudritz, R.E., Astrophys. J., 429, 177-191 (1994)

Helou, G., Lu, N.Y., Werner, M.W., Malhotra, S., Silbermann, N., Astrophys. J., 532, L21-L24 (2000)

Higdon, S.J.U., Armus, L., Higdon, J.L., Soifer, B.T., Spoon, H.W.W., Astrophys. J., 648, 323-339 (2006) 
Hills, J.G., Astrophys. J., 235, 986-991 (1980)

Ho, P.T.P., Beck, S.C., Turner, J.L., Astrophys. J., 349, 57-66 (1990)

Ho, L.C., Filippenko, A.V., Astrophys. J., 466, L83-L86 (1996a)

Ho, L.C., Filippenko, A.V., Astrophys. J., 472, 600-610 (1996b)

Hodge, P.W., Astrophys. J., 133, 413-419 (1961)

Hollenbach, D.J., Tielens, A.G.G.M., Reviews of Modern Physics, 71, 173-230 (1999)

Holtzman, J.A., et al., Astron. J., 103, 691-702 (1992)

Homeier, N.L., Alves, J., Astron. Astrophys., 430, 481-489 (2005)

Hughes, J.P., Rakowski, C.E., Burrows, D.N., Slane, P.O., Astrophys. J., 528, L109L113 (2000)

Hunter, D.A., Shaya, E.J., Holtzman, J.A., Light, R.M., O’Neil, E.J., Jr., Lynds, R., Astrophys. J., 448, 179-194 (1995)

Hunter, D.A., O’Neil, E.J., Jr., Lynds, R., Shaya, E.J., Groth, E.J., and Holtzman, J.A., Astrophys. J., 459, L27-L30 (1996)

Hunter, D.A., O'Connell, R.W., Gallagher, J.S., Smecker-Hane, T.A., Astron. J., 120, 2383-2401 (2000)

Israel, F.P., de Graauw, T., van de Stadt, H., de Vries, C.P., Astrophys. J., 303, 186197 (1986)

Johnson, K.E., Kobulnicky, H.A., Astrophys. J., 597, 923-928 (2003)

Johnson, K.E., Indebetouw, R., Pisano, D.J., Astron. J., 126, 101-112 (2003)

Karachentsev, I.D., et al. 2007, Astron. J., 133, 504-517 (2007)

Kaufman, M.J., Wolfire, M.G., Hollenbach, D.J., Astrophys. J., 644, 283-299 (2006)

Kaufman, M.J., Wolfire, M.G., Hollenbach, D.J., Luhman, M.L., Astrophys. J., 527, 795-813 (1999)

Kawara, K., Nishida, M., Phillips, M.M., Astrophys. J., 337, 230-235 (1989)

Kennicutt, R.C., Jr., Astrophys. J., 287, 116-130 (1984)

Kennicutt, R.C., Jr., Annu. Rev. Astron. Astrophys., 36, 189-232 (1998)

Kennicutt, R.C., Jr., Astrophys. J., 498, 541-552 (1998)

Kim, S.S., Figer, D.F., Lee, H.M., Morris, M., Astrophys. J., 545, 301-308 (2000)

Kim, S.S., Figer, D.F., Kudritzki, R.P., and Najarro, F., Journal of Korean Astronomical Society, 40, 153-155 (2007)

Kim, S.S., Figer, D.F., Morris, M., Astrophys. J., 607, L123-L126 (2004)

Kobulnicky, H.A., Johnson, K.E., Astrophys. J., 527, 154-166 (1999)

Kobulnicky, H.A., Skillman, E.D., Astron. J., 135, 527-537 (2008)

Koen, C., Mon. Not. R. Astron. Soc., 365, 590-594 (2006)

Kroupa, P., Mon. Not. R. Astron. Soc., 322, 231-246 (2001)

Kroupa, P., Boily, C.M., Mon. Not. R. Astron. Soc., 336, 1188-1194 (2002)

Krumholz, M.R., Matzner, C.D., McKee, C.F., Astrophys. J., 653, 361-382 (2006)

Krumholz, M.R., Tan, J.C., Astrophys. J., 654, 304-315 (2007)

Lada, C.J., Margulis, M., Dearborn, D., Astrophys. J., 285, 141-152 (1984)

Lada, C.J., Lada, E.A., Annu. Rev. Astron. Astrophys., 41, 57-115 (2003)

Lang, C.C., Goss, W.M., Morris, M., Astron. J., 121, 2681-2705 (2001)

Larsen, S.S., Origlia, L., Brodie, J., Gallagher, J.S., Mon. Not. R. Astron. Soc., 383, 263-276 (2008) 
Leitherer, C., et al., ApJS, 123, 3-40 (1999)

Lepp, S., Dalgarno, A., Astron. Astrophys., 306, L21-L24 (2006)

Lonsdale, C.J., et al., Publ. Astron. Soc. Pac., 115, 897-927 (2003)

Lutz, D., Kunze, D., Spoon, H.W.W., Thornley, M.D., Astron. Astrophys., 333, L75L78 (1998)

Lynds, C.R., Sandage, A.R., Astron. J., 68, 285 (1963)

Mackey, A.D., Gilmore, G.F., Mon. Not. R. Astron. Soc., 338, 85-119 (2003)

Maloney, P., Black, J.H., Astrophys. J., 325, 389-401 (1988)

Maloney, P.R., Hollenbach, D.J., Tielens, A.G.G.M., Astrophys. J., 466, 561-584 (1996)

Mangum, J.G., Darling, J., Menten, K.M., Henkel, C., Astrophys. J., 673, 832-846 (2008)

Maoz, D., Barth, A.J., Ho, L.C., Sternberg, A., Filippenko, A.V., Astron. J., 121, 3048-3074 (2001)

Maoz, D., Barth, A.J., Sternberg, A., Filippenko, A.V., Ho, L.C., Macchetto, F.D., Rix, H.-W., and Schneider, D.P., Astron. J., 111, 2248-2264 (1996)

Maoz, D., Filippenko, A.V., Ho, L.C., Macchetto, F.D., Rix, H.-W., Schneider, D.P., ApJS, 107, 215-226 (1996)

Martín, S., Martín-Pintado, J., Mauersberger, R., Astron. Astrophys., 450, L13-L16 (2006)

Martín, S., Mauersberger, R., Martín-Pintado, J., Henkel, C., and García-Burillo, S., ApJS, 164, 450-476 (2006)

Martín, S., Requena-Torres, M.A., Martín-Pintado, J., Mauersberger, R. 2008, ApSS, 313, 303-306 (2008)

Martín-Hernández, N.L., Schaerer, D., Sauvage, M., Astron. Astrophys., 429, 449467 (2005)

Martín-Hernández, N.L., Vermeij, R., Tielens, A.G.G.M., van der Hulst, J.M., Peeters, E., Astron. Astrophys., 389, 286-294 (2002)

Massey, P., Hunter, D.A., Astrophys. J., 493, 180 (1998)

Mathieu, R.D., Astrophys. J., 267, L97-L101 (1983)

Mauersberger, R., Henkel, C., Astron. Astrophys., 245, 457-466 (1991)

Mauersberger, R., Henkel, C., Weiß, A., Peck, A.B., Hagiwara, Y., Astron. Astrophys., 403, 561-561 (2003)

McCrady, N., Graham, J.R., Astrophys. J., 663, 844-856 (2007)

McCrady, N., Gilbert, A.M., Graham, J.R., Astrophys. J., 596, 240-252 (2003)

McCrady, N., Graham, J.R., Vacca, W.D., Astrophys. J., 621, 278-284 (2005)

McKee, C.F., Ostriker, E.C., Annu. Rev. Astron. Astrophys., 45, 565-687 (2007)

McLaughlin, D.E., Fall, S.M., Astrophys. J., 679, 1272-1287 (2008)

McLaughlin, D.E., van der Marel, R.P., ApJS, 161, 304-360 (2005)

McMillan, S.L.W., Portegies Zwart, S.F., Astrophys. J., 596, 314-322 (2003)

Meier, D.S., Turner, J.L., Beck, S.C., Astron. J., 122, 1770-1781 (2001)

Meier, D.S., Turner, J.L., Beck, S.C., Astron. J., 124, 877-855 (2002)

Meier, D.S., Turner, J.L., Astron. J., 127, 2069-2084 (2004)

Meier, D.S., Turner, J.L., Astrophys. J., 618, 259-280 (2005)

Meier, D.S., Turner, J.L., submitted (2008) 
Meier, D.S., Turner, J.L., Hurt, R.L., Astrophys. J., 675, 281-302 (2008)

Meijerink, R., Spaans, M., Astron. Astrophys., 436, 397-409 (2005)

Meijerink, R., Spaans, M., Israel, F.P., Astrophys. J., 650, L103-L106 (2006)

Melena, N.W., Massey, P., Morrell, N.I., Zangari, A.M., Astron. J., 135, 878-891 (2008)

Mengel, S., Lehnert, M.D., Thatte, N., Genzel, R., Astron. Astrophys., 443, 41-60 (2005)

Mengel, S., Tacconi-Garman, L.E., Astron. Astrophys., 466, 151-155 (2007)

Mengel, S., Tacconi-Garman, L.E., ArXiv e-prints, 803, arXiv:0803.4471 (2008)

Meurer, G.R., Heckman, T.M., Leitherer, C., Kinney, A., Robert, C., Garnett, D.R., Astron. J., 110, 2665-2691 (1995)

Meylan, G., The Globular Cluster-Galaxy Connection, 48, 588-600 (1993)

Mills, B.Y., Turtle, A.J., Watkinson, A., Mon. Not. R. Astron. Soc., 185, 263-276 (1978)

Mirabel, I.F., Sanders, D.B., Astrophys. J., 340, L53-56 (1989)

Mirabel, I.F., et al., Astron. Astrophys., 333, L1-L4 (1998)

Mohan, N.R., Anantharamaiah, K.R., Goss, W.M., Astrophys. J., 557, 659-670 (2001)

Morse, J.A., Davidson, K., Bally, J., Ebbets, D., Balick, B., Frank, A., Astron. J., 116, 2443-2461 (1998)

Myers, P.C., Astrophys. J., 270, 105-118 (1983)

Najarro, F., Figer, D.F., Ap\&SS, 263, 251-254 (1998)

Noyola, E., Gebhardt, K., Astron. J., 134, 912-925 (2007)

Nürnberger, D.E.A. 2004, Ph.D.Dissertation, Univ. Würzburg

Nürnberger, D.E.A., Bronfman, L., Yorke, H.W., Zinnecker, H., Astron. Astrophys., 394, 253-269 (2002)

Nürnberger, D.E.A., Petr-Gotzens, M.G., Astron. Astrophys., 382, 537-553 (2002)

O'Connell, R.W., Gallagher, J.S., III, and Hunter, D.A., Astrophys. J., 433, 65-69 (1994)

O’Connell, R.W., Gallagher, J.S., III, Hunter, D.A., Colley, W.N., Astrophys. J., 446, L1-L4 (1995)

Oey, M.S., Clarke, C.J., Astrophys. J., 620, L43-L46 (2005)

Omont, A., Reports of Progress in Physics, 70, 1099-1176 (2007)

Origlia, L., Leitherer, C., Aloisi, A., Greggio, L., Tosi, M., Astron. J., 122, 815-824 (2001)

Padoan, P., Nordlund, Å., Astrophys. J., 576, 870-879 (2002)

Padoan, P., Nordlund, Å., Kritsuk, A.G., Norman, M.L., Li, P.S., Astrophys. J., 661, 972-981 (2007)

Pandey, A.K., Ogura, K., Sekiguchi, K., PASJ, 52, 847-865 (2000)

Parmentier, G., Gilmore, G., Mon. Not. R. Astron. Soc., 377, 352-372 (2007)

Parmentier, G., Goodwin, S.P., Kroupa, P., Baumgardt, H., Astrophys. J., 678, 347 352 (2008)

Peeters, E., Spoon, H.W.W., Tielens, A.G.G.M., Astrophys. J., 613, 986-1003 (2004)

Rieke, G.H., Lebofsky, M.J., Astrophys. J., 220, L37-L41 (1978) 
Rieke, G.H., Lebofsky, M.J., Annu. Rev. Astron. Astrophys., 17, 477-511 (1979)

Rieke, G.H., Lebofsky, M.J., Thompson, R.I., Low, F.J., Tokunaga, A.T., Astrophys. J., 238, 24-40 (1980)

Rieke, G.H., Low, F.J., Astrophys. J., 197, 17-23

Rigby, J.R., Rieke, G.H., Astrophys. J., 606, 237-257 (2004)

Rodríguez-Rico, C.A., Goss, W.M., Turner, J.L., and Gómez, Y., Astrophys. J., 670, 295-300 (2007)

Rodríguez-Rico, C.A., Goss, W.M., Zhao, J.-H., Gómez, Y., Anantharamaiah, K.R., Astrophys. J., 644, 914-923 (2006)

Roy, A.L., Goss, W.M., Anantharamaiah, K.R., Astron. Astrophys., 483, 79-88 (2008)

Sakamoto, K., Scoville, N.Z., Yun, M.S., Crosas, M., Genzel, R., and Tacconi, L.J., Astrophys. J., 514, 68-76 (1999)

Sanders, D.B., Mirabel, I.F., Astrophys. J., 298, L31-L35 (1985)

Sanders, D.B., Mirabel, I.F., Annu. Rev. Astron. Astrophys., 34, 749-792 (1996)

Sanders, D.B., Mazzarella, J.M., Kim, D.-C., Surace, J.A., and Soifer, B.T., Astron. J., 126, 1607-1664 (2003)

Sanders, D.B., Scoville, N.Z., Solomon, P.M., Astrophys. J., 289, 373-387 (1985)

Sanders, D.B., Scoville, N.Z., Young, J.S., Soifer, B.T., Schloerb, F.P., Rice, W.L., and Danielson, G.E., Astrophys. J., 305, L45-L49 (1986)

Sanders, D.B., Soifer, B.T., Elias, J.H., Neugebauer, G., Matthews, K., Astrophys. J., 328, L35-L39 (1988)

Sargent, W.L.W., Searle, L., Astrophys. J., 162, L155-L160 (1970)

Saviane, I., Momany, Y., da Costa, G.S., Rich, R.M., Hibbard, J.E., Astrophys. J., 678, 179-186 (2008)

Scalo, J.M., Fundamentals of Cosmic Physics, 11, 1-278 (1986)

Scalo, J., The Stellar Initial Mass Function (38th Herstmonceux Conference), 142, 201 (1998)

Schaerer, D., Contini, T., Kunth, D., Meynet, G. 1997, Astrophys. J., 481, L75-L78

Schaerer, D., Contini, T., Kunth, D., Astron. Astrophys., 341, 399-417 (1999)

Schaerer, D., Contini, T., Pindao, M., A\&AS, 136, 35-52 (1999)

Schmidt, M., Astrophys. J., 129, 243-258 (1959)

Scoville, N.Z., Yun, M.S., Sanders, D.B., Clemens, D.P., and Waller, W.H., ApJS, 63, 821-915 (1987)

Shepherd, D.S., Kurtz, S.E., Astrophys. J., 523, 690-700 (1999)

Shin, J., Kim, S.S., Takahashi, K., Mon. Not. R. Astron. Soc., 386, L67-L71 (2008)

Shioya, Y., Taniguchi, Y., Trentham, N., Mon. Not. R. Astron. Soc., 321, 11-17 (2001)

Silich, S., Tenorio-Tagle, G., Muñoz-Tuñón, C., Astrophys. J., 669, 952-958 (2007)

Simon, M., Simon, T., Joyce, R.R., Astrophys. J., 227, $64-66$ (1979)

Sirianni, M., Nota, A., Leitherer, C., De Marchi, G., Clampin, M., Astrophys. J., 533, 203-214 (2000)

Smith, L.F., Mezger, P.G., Biermann, P., Astron. Astrophys., 66, 65-76 (1978)

Smith, L.J., Gallagher, J.S., Mon. Not. R. Astron. Soc., 326, 1027-1040 (2001) 
Smith, L.J., Westmoquette, M.S., Gallagher, J.S., O'Connell, R.W., Rosario, D.J., de Grijs, R., Mon. Not. R. Astron. Soc., 370, 513-527 (2006)

Soifer, B.T., et al., Astrophys. J., 283, L1-L4 (1984)

Soifer, B.T., Sanders, D.B., Neugebauer, G., Danielson, G.E., Lonsdale, C.J., Madore, B.F., Persson, S.E., Astrophys. J., 303, L41-L44 (1986)

Soifer, B.T., Neugebauer, G., Houck, J.R., Annu. Rev. Astron. Astrophys., 25, 187230 (1987)

Soifer, B.T., Sanders, D.B., Madore, B.F., Neugebauer, G., Danielson, G.E., Elias, J.H., Lonsdale, C.J., Rice, W.L., Astrophys. J., 320, 238-257 (1987)

Solomon, P.M., Downes, D., Radford, S.J.E., Barrett, J.W., Astrophys. J., 478, 144161 (1997)

Solomon, P.M., Rivolo, A.R., Barrett, J., Yahil, A., Astrophys. J., 319, 730-741 (1987)

Stacey, G.J., Geis, N., Genzel, R., Lugten, J.B., Poglitsch, A., Sternberg, A., \& Townes, C.H., Astrophys. J., 373, 423-444 (1991)

Stanford, S.A., Sargent, A.I., Sanders, D.B., Scoville, N.Z., Astrophys. J., 349, $492-$ 496 (1990)

Sternberg, A., Astrophys. J., 506, 721-726 (1998)

Stevens, I.R., Forbes, D.A., Norris, R.P., Mon. Not. R. Astron. Soc., 335, 1079-1084 (2002)

Stolte, A., Ph.D.Thesis, Combined Faculties for the Natural Sciences and for Mathematics of the Ruperto-Carola University of Heidelberg (2003)

Stolte, A., Brandner, W., Grebel, E.K., Lenzen, R., Lagrange, A.-M., Astrophys. J., 628, L113-L116 (2005)

Stolte, A., Grebel, E.K., Brandner, W., Figer, D.F., Astron. Astrophys., 394, 459-478 (2002)

Stolte, A., Brandner, W., Brandl, B., Zinnecker, H., Astron. J., 132, 253-270 (2006)

Strong, A.W., et al., Astron. Astrophys., 207, 1-15 (1988)

Strong, A.W., Moskalenko, I.V., Reimer, O., Digel, S., and Diehl, R., Astron. Astrophys., 422, L47-L50 (2004)

Sung, H., Bessell, M.S., Astron. J., 127, 1014-1028 (2004)

Tacconi, L.J., Young, J.S., Astrophys. J., 352, 595-604 (1990)

Tan, J.C., Krumholz, M.R., McKee, C.F., Astrophys. J., 641, L121-L124 (2006)

Thilker, D.A., et al., Astrophys. J., 619, L79-L82 (2005)

Thornley, M.D., Schreiber, N.M.F., Lutz, D., Genzel, R., Spoon, H.W.W., Kunze, D., Sternberg, A., Astrophys. J., 539, 641-657 (2000)

Tielens, A.G.G.M., The Physics and Chemistry of the Interstellar Medium, by A.G.G.M.Tielens, pp..ISBN 0521826349.Cambridge, UK: Cambridge (2005) University Press, 2005

Tielens, A.G.G.M., Hollenbach, D., Astrophys. J., 291, $722-754$ (1985)

Tielens, A.G.G.M., Hollenbach, D., Astrophys. J., 291, 747-754 (1985)

Tilanus, R.P.J., Allen, R.J., Astron. Astrophys., 274, 707-729 (1993)

Tremonti, C.A., Calzetti, D., Leitherer, C., Heckman, T.M., Astrophys. J., 555, 322$337(2001)$ 
Tsai, C.-W., Turner, J.L., Beck, S.C., Crosthwaite, L.P., Ho, P.T.P., Meier, D.S., Astron. J., 132, 2383-2397 (2006)

Turner, J.L., Beck, S.C., Astrophys. J., 602, L85-L88 (2004)

Turner, J.L., Ho, P.T.P., Astrophys. J., 421, 122 (1994)

Turner, J.L., Beck, S.C., Crosthwaite, L.P., Larkin, J.E., McLean, I.S., Meier, D.S., Nature, 423, 621-623 (2003)

Turner, J.L., Beck, S.C., Ho, P.T.P., Astrophys. J., 532, L109-L112 (2000)

Turner, J.L., Beck, S.C., Hurt, R.L., Astrophys. J., 474, L11-L14 (1997)

Turner, J.L., Ho, P.T.P., Beck, S.C., Astron. J., 116, 1212-1220 (1998)

Vacca, W.D., Conti, P.S., Astrophys. J., 401, 543-558 (1992)

van Dishoeck, E.F., Blake, G.A., Annu. Rev. Astron. Astrophys., 36, 317-368 (1998)

Vanzi, L., Sauvage, M., Astron. Astrophys., 415, 509-520 (2004)

Vázquez, G.A., Leitherer, C., Astrophys. J., 621, 695-717 (2005)

Vázquez, G.A., Leitherer, C., Heckman, T.M., Lennon, D.J., de Mello, D.F., Meurer, G.R., Martin, C.L., Astrophys. J., 600, 162-181 (2004)

Veilleux, S., Cecil, G., Bland-Hawthorn, J., Annu. Rev. Astron. Astrophys., 43, 769826 (2005)

Walborn, N.R., et al., Astron. J., 123, 2754-2771 (2002)

Watson, A.M., et al., Astron. J., 112, 534-544 (1996)

Weedman, D.W., Feldman, F.R., Balzano, V.A., Ramsey, L.W., Sramek, R.A., Wuu, C.-C., Astrophys. J., 248, 105-112 (1981)

Weidner, C., Kroupa, P., Mon. Not. R. Astron. Soc., 348, 187-191 (2004)

Weigelt, G., Baier, G., Astron. Astrophys., 150, L18-L20 (1985)

Welch, W.J., Dreher, J.W., Jackson, J.M., Terebey, S., Vogel, S.N., Science, 238, 1550-1555 (1987)

Whitmore, B.C., Schweizer, F., Astron. J., 109, 960-980 (1995)

Whitmore, B.C., Schweizer, F., Leitherer, C., Borne, K., Robert, C., Astron. J., 106, 1354-1370 (1993)

Whitmore, B.C., Zhang, Q., Leitherer, C., Fall, S.M., Schweizer, F., Miller, B.W., Astron. J., 118, 1551-1576 (1999)

Willner, S.P., Soifer, B.T., Russell, R.W., Joyce, R.R., Gillett, F.C., Astrophys. J., 217, L121-L124 (1977)

Wilson, C.D., Scoville, N., Madden, S.C., Charmandaris, V., Astrophys. J., 542, 120-127 (2000)

Wilson, C.D., Scoville, N., Madden, S.C., Charmandaris, V., Astrophys. J., 599, 1049-1066 (2003)

Wolfire, M.G., Hollenbach, D., Tielens, A.G.G.M., Astrophys. J., 402, 195-215 (1993)

Wong, T., Blitz, L., Astrophys. J., 569, 157-183 (2002)

Wood, D.O.S., Churchwell, E. 1989, ApJS, 69, 831-895 (1989)

Young, J.S., Allen, L., Kenney, J.D.P., Lesser, A., Rownd, B. 1996, Astron. J., 112, 1903-1927 (1996)

Young, J.S., Kenney, J.D., Tacconi, L., Claussen, M.J., Huang, Y.-L., TacconiGarman, L., Xie, S., Schloerb, F.P., Astrophys. J., 311, L17-L21 (1986)

Young, J.S., Scoville, N.Z., Annu. Rev. Astron. Astrophys., 29, 581-625 (1991) 
Young, J.S., Schloerb, F.P., Kenney, J.D., Lord, S.D., Astrophys. J., 304, 443-458 (1986)

Yun, M.S., Ho, P.T.P., Lo, K.Y., Nature, 372, 530-532 (1994)

Yungelson, L.R., van den Heuvel, E.P.J., Vink, J.S., Portegies Zwart, S.F., de Koter, A., Astron. Astrophys., 477, 223-237 (2008)

Zhang, Q., Fall, S.M., Astrophys. J., 527, L81-L84 (1999)

Zinnecker, H., Yorke, H.W., Annu. Rev. Astron. Astrophys., 45, 481-463 (2007) 